\title{
Characterization and error analysis of an $N \times N$ unfolding procedure applied to filtered, photoelectric x-ray detector arrays. II. Error analysis and generalization
}

\author{
D. L. Fehl, G. A. Chandler, W. A. Stygar, R. E. Olson, C. L. Ruiz, J. J. Hohlfelder, L. P. Mix, and F. Biggs \\ Sandia National Laboratories, Albuquerque, New Mexico 87185, USA
}

M. Berninger, P. O. Frederickson, and R. Frederickson

National Security Technologies, LLC, Los Alamos, New Mexico 87544, USA

(Received 28 April 2010; published 30 December 2010)

\begin{abstract}
A five-channel, filtered-x-ray-detector (XRD) array has been used to measure time-dependent, soft$x$-ray flux emitted by $z$-pinch plasmas at the $Z$ pulsed-power accelerator (Sandia National Laboratories, Albuquerque, New Mexico, USA). The preceding, companion paper [D. L. Fehl et al., Phys. Rev. ST Accel. Beams 13, 120402 (2010)] describes an algorithm for spectral reconstructions (unfolds) and spectrally integrated flux estimates from data obtained by this instrument. The unfolded spectrum $S_{\text {unfold }}(E, t)$ is based on $(N=5)$ first-order B-splines (histograms) in contiguous unfold bins $j=1, \ldots, N$; the recovered x-ray flux $\mathcal{F}_{\text {unfold }}(t)$ is estimated as $\int S_{\text {unfold }}(E, t) d E$, where $E$ is x-ray energy and $t$ is time. This paper adds two major improvements to the preceding unfold analysis: (a) Error analysis.-Both data noise and response-function uncertainties are propagated into $S_{\text {unfold }}(E, t)$ and $\mathcal{F}_{\text {unfold }}(t)$. Noise factors $\nu$ are derived from simulations to quantify algorithm-induced changes in the noise-to-signal ratio (NSR) for $S_{\text {unfold }}$ in each unfold bin $j$ and for $\mathcal{F}_{\text {unfold }}\left(\nu \equiv N S R_{\text {output }} / N S R_{\text {input }}\right)$ : for $S_{\text {unfold }}, 1 \lesssim \nu_{j} \lesssim 30$, an outcome that is strongly spectrally dependent; for $\mathcal{F}_{\text {unfold }}, 0.6 \lesssim \nu_{\mathcal{F}} \lesssim 1$, a result that is less spectrally sensitive and corroborated independently. For nominal $z$-pinch experiments, the combined uncertainty (noise and calibrations) in $\mathcal{F}_{\text {unfold }}(t)$ at peak is estimated to be $\sim 15 \%$. (b) Generalization of the unfold method.-Spectral sensitivities (called here passband functions) are constructed for $S_{\text {unfold }}$ and $\mathcal{F}_{\text {unfold }}$. Predicting how the unfold algorithm reconstructs arbitrary spectra is thereby reduced to quadratures. These tools allow one to understand and quantitatively predict algorithmic distortions (including negative artifacts), to identify potentially troublesome spectra, and to design more useful response functions.
\end{abstract}

DOI: 10.1103/PhysRevSTAB.13.120403

PACS numbers: 52.70.La, 52.59.Qy, 52.58.Lq, 52.50.-b

\section{INTRODUCTION: A SUMMARY OF PRINCIPAL EQUATIONS FOR THE UNFOLD ALGORITHM IN PART 1}

Part 1 of this article formulated and tested a spectral unfold algorithm, applied to experimental data $D_{i}(t)$ from a filtered-x-ray-detector (XRD) diagnostic of $N$ channels (typically, $N=5$ ). In this method, the channel-wise response functions $R_{i}(E)$ are assumed to be calibrated, and the incident x-ray spectrum $S(E, t)$ under diagnosis is presumed to be at least piecewise continuous-though not necessarily Planckian, $S_{b b}(E, T)$. A priori information about the source is used to formulate a reconstruction $S_{\text {unfold }}(E, t)$ of $S(E, t)$, where $S_{\text {unfold }}$ is represented by an $N$-bin histogram over an unfold interval, $[\Delta E]=$ $\left[E_{\mathrm{LO}}, E_{\mathrm{HI}}\right]$ (Pt. 1: Sec. III and Pt. 1: Table II). The diagnostic goal is to estimate the incident X-ray flux $\mathcal{F}_{[\Delta E]}(t)$ in $[\Delta E]$ from the spectral integral $\mathcal{F}_{\text {unfold }}(t)$ of $S_{\text {unfold }}(E, t)$. [Equations (1)-(6) below summarize this algorithm.] In part 1 the unfold method was tested in noise-free simulations based on prescribed incident spectra $S(E, t)$ : binwise averages, $\langle S\rangle_{j}$, were compared to the corresponding histogram values $S_{j}(t)$ (also called unfold coefficients), as were the flux values, $\mathcal{F}_{[\Delta E]}(t)$ and $\mathcal{F}_{\text {unfold }}(t)$. Further tests compared the results of this algorithm with other methods for simulated and real data.

In this second part of the article, we extend the characterization of the unfold algorithm. Section II examines the effect of perturbations $\varepsilon_{i}$ [Eq. (1)] on the reconstruction $S_{\text {unfold }}$. The propagation of both systematic errors and random uncertainties are considered for input data noise, calibrational uncertainties in the response functions, and real data. (Here, the terms systematic error, deterministic error, and bias are considered equivalent.) Section III generalizes the histogram unfold algorithm so that spectral reconstruction for arbitrary incident spectral functions is reduced to quadratures, an approach that makes several otherwise difficult issues accessible. For example, one can now address the general quantitative validity of the unfold algorithm as well as its specific behavior in the simulations described in part 1. Neither analysis (Secs. II and III) has previously been reported for the $Z$ diagnostic nor, we believe, for similarly filtered-detector arrays. A summary and concluding remarks appear in Sec. IV. Several derivations and comments to the main text are given in five appendices and endnotes. References to part 1 of this 
article are prefixed with "Pt. 1:" (e.g., "cf. Pt. 1: Fig. 2"); references to this part contain no prefix (e.g., "cf. Fig. 1").

For convenience here, the unfold algorithm is summarized as follows:

$$
\begin{aligned}
\int_{0}^{E_{\mathrm{MAX}}} R_{i}(E) S_{\text {unfold }}(E, t) d E \\
\quad \equiv \int_{0}^{E_{\mathrm{MAX}}} R_{i}(E) S(E, t) d E+\varepsilon_{i}(t) \\
\quad \equiv d_{i}(t)+\varepsilon_{i}(t) \equiv D_{i}(t) \quad(i=1, \ldots, N)
\end{aligned}
$$

is the data model: $S(E, t)$ is the incident spectrum; $\left\{R_{i}(E)\right\}_{i=1}^{N}$ is the calibrated set of response functions; $d_{i}(t)$ is the noise-free x-ray channel data linked to both $R_{i}$ and $S ; \varepsilon_{i}(t)$ denotes uncertainties and noise; and $D_{i}(t)$
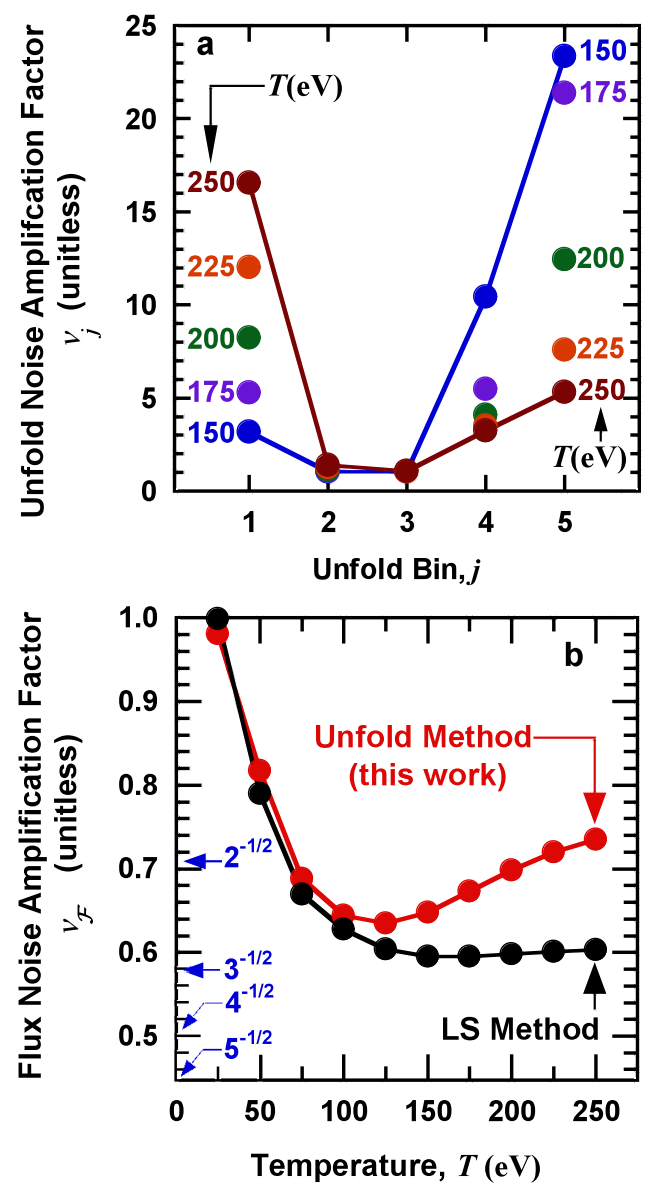

FIG. 1. Noise amplification in the unfolding process for uniform relative data errors. Part (a) gives the binwise noise factor (NF) $\nu_{j}[T]$-i.e., $N S R\left(S_{j}\right) / N S R\left(D_{i}\right)$-for Planckian spectra, $T=150-250 \mathrm{eV}$. (The colored, broken lines indicate the envelope of these calculations.) Part (b) shows the corresponding NF's $\nu_{\mathcal{F}}[T]$-i.e., $N S R\left(\mathcal{F}_{\text {unfold }}\right) / N S R\left(D_{i}\right)$ and $N S R\left(\mathcal{F}_{L S}\right) /$ $N S R\left(D_{i}\right)$-for the unfolded flux estimate $\mathcal{F}_{\text {unfold }}$ (red curve) and for a different flux estimate $\mathcal{F}_{L S}$ [Ref. [8]] (Planckian spectra, $T=25-250 \mathrm{eV})$. The sequence of numbers $m^{-(1 / 2)}$ $(m=1, \ldots, 5)$ on the left-hand scale represent hypothetical degrees of freedom for noise averaging. thus represents experimental data. $E_{\mathrm{MAX}}$ is an x-ray energy beyond which $R_{i}$ and $S$ are disregarded (Pt. 1: Sec. II A).

The unfolded reconstruction $S_{\text {unfold }}(E, t)$, given $D_{i}(t)$, is then

$$
\begin{aligned}
S_{\text {unfold }}(E, t) & =\sum_{j=1}^{N} S_{j}(t) B_{j}(E) \\
& =\sum_{j=1}^{N}\left[\sum_{i=1}^{N}\left(\mathbb{R}^{-1}\right)_{j i} D_{i}(t)\right] B_{j}(E),
\end{aligned}
$$

where the $B_{j}$ 's are first-order B-splines (histograms) that divide the unfold interval $[\Delta E] \subseteq\left[0, E_{\mathrm{MAX}}\right]$ into $N$ unfold bins $\left[\Delta E_{j}\right]$, and where

$$
(\mathbb{R})_{i j}=\int_{0}^{E_{\mathrm{MAX}}} R_{i}(E) B_{j}(E) d E=\int_{E_{j}}^{E_{j+1}} R_{i}(E) d E
$$

[Pt. 1: Eqs. (12) and (13), Table II]. Existence and uniqueness in Eq. (2) depend on the condition of $\mathbb{R}$, as previously verified [Pt. 1: Eqs. (24) and (25)]. The quantities, $S_{j}(t)$, $D_{i}(t), d_{i}(t)$, and $\varepsilon_{i}(t)$, above can formally be represented by $N$-dimensional vectors: $\mathbf{S}(t), \mathbf{D}(t), \mathbf{d}(t)$, and $\mathbf{\varepsilon}(t)$. Equation (2) is then equivalent to

$$
S_{j}(t)=\left[\mathbb{R}^{-1} \mathbf{D}(t)\right]_{j}
$$

[Pt. 1: Eq. (21)]. Finally, given $S_{\text {unfold }}(E, t)$, the unfolded $\mathrm{x}$-ray flux is

$$
\mathcal{F}_{\text {unfold }} \equiv \Delta \mathbf{E} \cdot \mathbf{S}(t) \equiv \sum_{j=1}^{N} \sum_{i=1}^{N}\left[\left(\mathbb{R}^{-1}\right)_{j i} D_{i}(t)\right] \Delta E_{j},
$$

which is, in general, time dependent [Pt. 1: Eq. (23)].

In noise-free simulations, the spectrum $S$ is specified. The unfolded values $S_{j}$ are then compared with the average values, $\langle S\rangle_{j}=\left(\Delta E_{j}\right)^{-1} \int_{\left[\Delta E_{j}\right]} S(E, t) d E$, of $S$ in each unfold bin $\left[\Delta E_{j}\right]$, and $\mathcal{F}_{\text {unfold }}$ is compared to $\mathcal{F}_{[\Delta E]}$ :

$$
\mathcal{F}_{[\Delta E]}(t) \equiv \int_{E_{\mathrm{LO}}}^{E_{\mathrm{HI}}} S(E, t) d E=\sum_{j=1}^{N}\left(\Delta E_{j}\right)\langle S\rangle_{j}(t) .
$$

Equations (1)-(6) have been formally written for physically admissible spectra $S(E)$ emitted by a $z$ pinch (i.e., conforming to the assumptions in Pt. 1: Table I), and are thusly used in Sec. II below. But, the same equations apply equally for more general "spectral functions," denoted by $f(E)$, and are used in Sec. III. Table I summarizes the mathematical definitions and notation used in both papers.

\section{UNCERTAINTY ANALYSIS}

Part 1 (Sec. IV) assessed the histogram algorithm under the best unfold conditions. The channel data and response functions were noise free, and identical responses were used for simulation and spectral reconstruction. This section relaxes these conditions by allowing uncertainty and inconsistency in both the $D_{i}$ 's and $R(E)_{i}$ 's. Such effects are 
TABLE I. Definitions and mathematical notations. All mathematical quantities considered in this article are real valued.

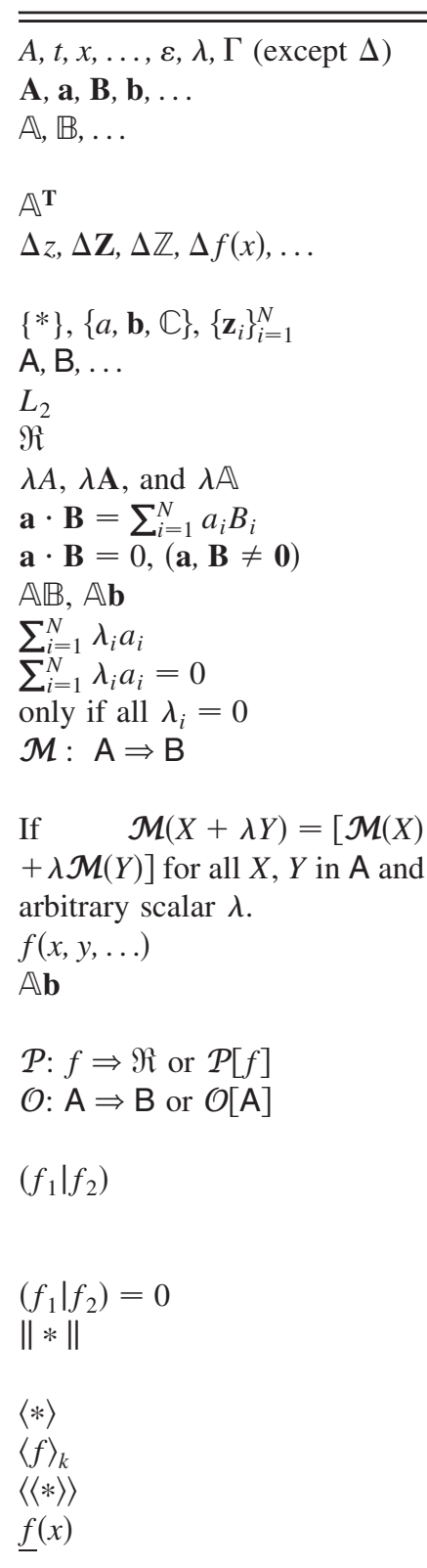

Random variable $a$ with distribution $N\left(0, \sigma^{2}\right)$

$\operatorname{cov}(a, b)$

Random vector $\mathbf{B}$

$\mathbb{C}(\mathbf{B})$

Spectra vs spectral functions:

$S(E), S(E, t)$

$f(E), f(E, t)$

$S_{b b}(E, T)$

\section{Scalars}

Vectors, defined by indexed components $A_{i}$ or as $N$-tuples $\left(A_{1}, \ldots, A_{N}\right)$

Matrices, defined by doubly indexed elements $A_{i j}$ or a two-dimensional array [e.g., Pt. 1, Eq. (24)].

Transpose of $\mathbb{A}$ : i.e., $\left(\mathbb{A}^{\mathbf{T}}\right)_{i j}=(\mathbb{A})_{j i}$

Differences in $z, \mathbf{Z}, \mathbb{Z}, f(x), \ldots$, relative to references $z_{\text {ref }}, \mathbf{Z}_{\text {ref }}, \mathbb{Z}_{\text {ref }}, f_{\text {ref }}(x) \ldots[\Delta f(x) \equiv$ $\left.f(x)-f_{\text {ref }}(x)\right]$

A set of objects (scalars, functions, vectors, matrices).

Names of sets

Hilbert Space of square-integrable functions over a specified domain.

The set of real numbers.

Scalar multiplication.

Inner product of vectors relative to an orthonormal basis.

Orthogonal vectors

Matrix-matrix and matrix-vector multiplication

A linear combination of objects (scalars, vectors, functions, etc.)

The objects $a_{1}, \ldots, a_{N}$ are linearly independent, except for functions evaluated at a finite number of points (i.e. a set of measure zero).

A mapping $\mathcal{M}$ between sets $A$ and $B$ associates each element in $A$ with exactly one element of B.

A linear mapping

A function maps a multidimensional set of variables $x, y, \ldots$ into $\mathfrak{R}$

A linear transformation between two vector sets by means of a matrix $\mathbb{A}$ associates each vector $\mathbf{b}$ in the first set with $\mathbf{A} \mathbf{b}$ in the second set

A functional, i.e., a mapping $\mathcal{P}$ from a set of functions $\{f(x)\}$ into $\mathfrak{i}$

An operator over $\mathrm{A}$, i.e., a mapping $\mathcal{O}$ of a set of functions $\mathrm{A}=\{A(x)\}$ into another set of functions $\mathrm{B}=\{B(x)\}$

The inner product, $\int_{0}^{E_{\mathrm{MAX}}} f_{1}(E) f_{2}(E) d E$, of two real-valued, spectral functions $f_{1}$ and $f_{2}$. Riemann integration is implied. (Functions requiring Lebesgue rules are not used in this article.)

Orthogonal functions if $f_{1}, f_{2} \neq 0$ for all $x$.

Norms: $\|a\|=|a|$ (the absolute value), $\|\mathbf{b}\|=\left[\sum_{i=1}^{N} b_{i}^{2}\right]^{1 / 2}$ (the Euclidian norm), and $\|f\|=$ $\sqrt{(f \mid f)}$.

An average over some set

The average of $f(E)$ over $\left[E_{k}, E_{k+1}\right]$

The successive (double) average $\langle[\langle *\rangle]\rangle$ over two sets.

The shape of a function $f(x)$ over $[a, b]: \underline{f}(x) \equiv f(x) / \int_{a}^{b} f\left(x^{\prime}\right) d x^{\prime}$ if $f(x)$ is non-negative, not identically zero, and integrable in $[a, b]$

A random variable assumed drawn from a normal distribution with specified distribution zero mean and variance $\sigma^{2}(a)=\left\langle(a-\langle a\rangle)^{2}\right\rangle=\left\langle a^{2}\right\rangle-\langle a\rangle^{2}$. The square root, $\sigma(a)$, of the variance is termed the standard deviation of the distribution.

The covariance, $\langle(a-\langle a\rangle)(b-\langle b\rangle)\rangle=\langle a b\rangle-\langle a\rangle\langle b\rangle$ between two random variables $a, b$. Such variables are correlated if $\operatorname{cov}(a, b) \neq 0$. If $a, b$ are statistically independent, then $\operatorname{cov}(a, b)=0$ (Ref. [1]) $\operatorname{cov}(a, a)=\sigma^{2}(a)$.

A vector with random components $\left(B_{1}, \ldots, B_{N}\right)$ which may, in general, be correlated.

The covariance matrix of a random vector $B$, an $N \times N$ array of elements, $[\mathbb{C}(\mathbf{B})]_{i j}=$ $\operatorname{cov}\left(B_{i}, B_{j}\right)=\operatorname{cov}\left(B_{j}, B_{i}\right)=[\mathbb{C}(\mathbf{B})]_{j i}$

Spectra, i.e., physically admissible functions of x-ray energy $E$ that satisfy the assumptions of Pt. 1: Table I and are associated with experimental data. $S(E, t)$ indicates a time-indexed sequence of such spectra.

Spectral functions, i.e., general members of $\mathrm{L}_{2}\left[0, E_{\mathrm{MAX}}\right]$, including negative-valued functions. Planckian functions, $S_{b b}(E, T)$ [Pt. 1, Eq. (7)].

$S(E), S_{b b}(E, T), f(E)$ are all elements of $\mathrm{L}_{2}\left[0, E_{\mathrm{MAX}}\right]$. 
TABLE I. (Continued)

\begin{tabular}{ll}
\hline \hline Associated functions: & \\
$S(E), f(E)$ & A spectrum or spectral function that generates channel data \\
$\langle S\rangle_{k},\langle f\rangle_{k}$ & The average of $S(E), f(E)$ in bin $\left[E_{k}, E_{k+1}\right]$ \\
$\Delta S_{B}(E), \Delta f_{B}(E)$ & The part of $S(E), f(E)$ not approximated by basis functions: e.g., $f(E)-\sum_{j=1}^{N}\langle f\rangle_{j} B_{j}(E)$ \\
$S_{\text {unfold }}(E), f_{\text {unfold }}(E)$ & The reconstructed spectrum obtained via Pt. 1, Eq. (21): $\sum_{j=1}^{N} S_{j} B_{j}(E), \sum_{j=1}^{N} f_{j} B_{j}(E)$ \\
$S_{j}, \mathbf{S}, f_{j}, \mathbf{f}$ & The unfold coefficients of $S_{\text {unfold }}(E), f_{\text {unfold }}(E)$, also denoted as the $N$ components of $\mathbf{S}, \mathbf{f}$. \\
\hline X-ray flux definitions: & \\
$\mathcal{F}$ & The "total" flux integral, $\int S(E) d E$ or $\int f(E) d E$, over $\left[0, E_{\mathrm{MAX}}\right]$. \\
$\mathcal{F}_{[\Delta E]}$ & The flux integral over $[\Delta E]=\left[E_{\mathrm{LO}}, E_{\mathrm{HI}}\right] \subseteq\left[0, E_{\mathrm{MAX}}\right]$. \\
$\mathcal{F}_{\text {unfold }}$ & The flux estimate from $S_{\text {unfold }}(E), f_{\text {unfold }}(E)$ over $[\Delta E]$ via Pt. 1, Eq. (23) \\
\hline \hline
\end{tabular}

assumed here to be independent and additive perturbations $[2,3]$ to the X-ray detection process, and $\varepsilon_{i}$ represents their sum in the data model [Eq. (1)]. Included in $\varepsilon_{i}$ are bias and random perturbations, which may be channel dependent and differ in time scale. Here, a general expression for the effect of these perturbations on the unfolded spectrum is first derived and then applied to several error models. Uncertainties are then estimated for real experimental data, and the section concludes with a comparison of simultaneously fielded, independent, filtered-XRD arrays at the $Z$ accelerator.

\section{A. A General formalism for error propagation in the $\boldsymbol{Z}$ unfold problem}

The recorded data $D_{i}$ in Eq. (1) are assumed due to an x-ray interaction term, $d_{i}=\int_{0}^{E_{\mathrm{MAX}}} R_{i}(E) S(E) d E$, plus perturbations $\varepsilon_{i}$, which can enter in two ways. Some perturbations $\Delta D_{i}$ superpose onto $d_{i}$ in the data-gathering process: e.g., signal noise, digitization errors, uncertainties in geometric factors, etc. Other perturbations add an energy-dependent factor $\Delta R_{i}(E)$ to the experimentally measured response functions $R_{i}(E)$ : e.g., the $\Delta R_{i}$ may result from drift and uncertainty in the fit parameters, $A_{i l}$ and $\tau_{i m}$ [Pt. 1: Appendix B, Eqs. (B1) and (B2)]. Such perturbations shift the matrix, $\mathbb{R} \rightarrow \mathbb{R}+\Delta \mathbb{R}$, in Eq. (3): i.e., $R_{i j} \rightarrow R_{i j}+\Delta R_{i j}$, where

$$
(\Delta \mathbb{R})_{i j}=\Delta R_{i j}=\int_{0}^{E_{\mathrm{MAX}}}\left[\Delta R_{i}(E)\right] B_{j}(E) d E .
$$

By whichever route, perturbations $\varepsilon_{i}$ to Eq. (1) induce deviations $\Delta S_{\text {unfold }}(E)$ in the nominally unfolded spectrum $S_{\text {unfold }}(E)$. The binwise effect $\Delta S_{j}$ on the nominal unfold coefficient $S_{j}$ is found (Appendix A) from Eq. (4) in vector-matrix notation:

$$
\Delta \mathbf{S}=\mathbb{R}^{-1}[\Delta \mathbf{D}+(\Delta \mathbb{R}) \mathbf{S}],
$$

where $(\Delta \mathbb{R}) \mathbf{S}$ is analogous to $\Delta \mathbf{D}$. Equation (8) is thus identical in form to the unfold algorithm [Eq. (4)]. Equation (2) then yields $\Delta S_{\text {unfold }}(E)$, with $\Delta S_{j}$ substituted for $S_{j}$. (General mathematical bounds for this process were noted in Pt. 1: Sec. III G.) This approach obviates the need for calculating $\Delta\left(\mathbb{R}^{-1}\right)$ when error propagation is attempted directly with Eq. (2).

An estimate of the perturbation $\Delta \mathcal{F}_{\text {unfold }}$ in $\mathcal{F}_{\text {unfold }}$ due to data and calibrational perturbations then follows (Appendix A) from Eq. (5):

$$
\Delta \mathcal{F}_{\text {unfold }}=\Delta \mathbf{E} \cdot\left[\left(\mathbb{R}^{-\mathbf{1}}\right)\{(\Delta \mathbf{D})+(\Delta \mathbb{R}) \mathbf{S}\}\right],
$$

where the components of $\Delta \mathbf{E}$ are the fixed unfold bin widths $\Delta E_{j}$ (Pt. 1: Table II).

Since data and calibrational perturbations have been assumed independent of one another, their effects may be treated separately and later combined. The rest of this section is devoted to examples of deterministic and random models for $\Delta \mathbf{D}$ and $\Delta \mathbb{R}$. In each case it is assumed that nominal values of $S_{j}, \mathbf{S}, S_{\text {unfold }}(E)$, and $\mathcal{F}_{\text {unfold }}$ have already been obtained from Eqs. (2)-(5).

\section{B. Deterministic effects (bias)}

If $\Delta \mathbf{D}$ and $\Delta \mathbb{R}$ represent deterministic effects, then $\Delta \mathbf{S}$ and $\Delta \mathcal{F}_{\text {unfold }}$ are the corresponding induced biases in nominal values of $\mathbf{S}$ and $\mathcal{F}_{\text {unfold }}$. Two examples show how the analysis of such perturbations establishes tolerances for the $Z$ diagnostic.

\section{Example: Data shifts $\Delta D_{i}$ alone}

Consider the recording of time-dependent, channel data by a set of waveform digitizers. Zero voltage for any diagnostic signal may or may not coincide with the "zero" digitization level (baseline) of the recording system. What relative errors in the nominal $\mathbf{S}$ and $\mathcal{F}_{\text {unfold }}$ can one expect from uncorrected baseline biases, $\Delta \mathbf{D}$ ?

We assume the following simplified model of the recording process: (a) The x-ray data $d_{i}(t)$ are assumed noise free and non-negative (relative to the actual zero voltage), and for convenience all the $d_{i}(t)$ 's peak at $t=t_{\text {peak }}$. (b) The recorded data, $D_{i}(t)=d_{i}(t)+\Delta D_{i}$, have a time-independent offset $\Delta D_{i}=\beta d_{i}\left(t_{\text {peak }}\right)$, where $|\beta| \ll$ 1 and is independent of channel. (c) $\mathcal{F}_{\text {unfold }}\left(t_{\text {peak }}\right) \equiv$ $\max \left[\mathcal{F}_{\text {unfold }}(t)\right]>0$.

The induced effects of $\Delta D_{i}$ in this model follow from Eqs. (8) and (9). In particular, the unfold coefficient bias, 
$\Delta \mathbf{S}=\beta \mathbf{S}\left(t_{\text {peak }}\right), \quad$ and the flux bias, $\Delta \mathcal{F}_{\text {unfold }}=$ $\beta \mathcal{F}_{\text {unfold }}\left(t_{\text {peak }}\right)$, are independent of time; but, the relative error, $\quad \Delta \mathcal{F}_{\text {unfold }}\left[\mathcal{F}_{\text {unfold }}(t)\right]^{-1}=\beta \mathcal{F}_{\text {unfold }}\left(t_{\text {peak }}\right) \times$ $\left[\mathcal{F}_{\text {unfold }}(t)\right]^{-1}$, is time dependent. Thus, if a relative bias, $\left|\Delta \mathcal{F}_{\text {unfold }}\right|\left[\mathcal{F}_{\text {unfold }}(t)\right]^{-1}$, as large as $10 \%$ is allowed for flux measurements in the range $0.1 \leq \mathcal{F}_{\text {unfold }}(t) \times$ $\left[\mathcal{F}_{\text {unfold }}\left(t_{\text {peak }}\right)\right]^{-1} \leq 1$, then the tolerance in the baseline bias must be no more than $1 \%$ of peak signal.

\section{Example: Calibrational shifts $\Delta R_{i}(E)$ alone}

Slow drifts in the XRD sensitivity $\chi_{i}(E)$ [Pt. 1: Eqs. (2) and (B1)] modify the response functions of a filtered-XRD array [4] and are an insidious perturbation to spectral reconstructions, if not corrected. The typical case involves channel data garnered from drifted responses, $R_{i}(E)+$ $\Delta R_{i}(E)$, but (inadvertently) unfolded with an earlier calibration $R_{i}(E)$. This problem can again be addressed with Eqs. (8) and (9), but an example has already been noted in Pt. 1: Fig. 10(a), where this scenario is labeled as "inconsistent." Specifically, noise-free channel data were simulated from $S_{b b}(E, 200 \mathrm{eV})$ and responses $R_{1 a}, \ldots, R_{5 a}$ (Pt. 1: Fig. 2) but unfolded with the (biased) inverse matrix $\mathbb{R}^{-1}$ from responses $R_{1}, \ldots, R_{5}$ (Pt. 1: Fig. 2). The unfolded spectrum is spurious, disagreeing with the "consistent" unfold (i.e., inverted with $\mathbb{R}_{a}^{-1}$ ) by at least $40 \%$ and flux estimate by $\sim 50 \%$. One can argue that spectral differences between the response sets $R_{1}, \ldots, R_{5}$ and $R_{1 a}, \ldots, R_{5 a}$ have been magnified by unfold-matrix condition number $\sim 11$ (cf. Pt. 1: Sec. III G). Potential errors of this size argue for frequent calibrations, modeling of calibrational drifts in the fielded environment, and multiple flux diagnostics.

\section{Random effects}

Random perturbations, $\Delta D_{i}$ and $\Delta R_{i j}$, induce random uncertainties in $\Delta S_{j}$ and $\Delta \mathcal{F}_{\text {unfold }}$ via the unfold algorithm. These terms are now represented by probability distributions [1], which for convenience we assume to be normal with zero means but nonzero variances-denoted $N\left(0, \sigma^{2}\left[\Delta D_{i}\right]\right)$ and $N\left(0, \sigma^{2}\left[\Delta R_{i j}\right]\right)$ [5]. Since Eqs. (8) and (9) are linear, $\Delta S_{j}$ and $\Delta \mathcal{F}_{\text {unfold }}$ are likewise distributed as $N\left(0, \sigma^{2}[\Delta S]\right)$ and $N\left(0, \sigma^{2}\left[\Delta \mathcal{F}_{\text {unfold }}\right]\right)$. The goal is to derive $\sigma^{2}\left(\Delta S_{j}\right)$ and $\sigma^{2}\left(\Delta \mathcal{F}_{\text {unfold }}\right)$ from $\sigma^{2}\left(\Delta D_{i}\right)$ and $\sigma^{2}\left(\Delta R_{i j}\right)$.

In the error propagation analysis here, statistical correlations [Table I] among $\Delta D_{i}, \Delta R_{i j}$, and $\Delta S_{j}$ must be considered: the $\Delta R_{i j}$ 's share the same measured fit parameters for response function $R_{i}(E)$ (Pt. 1: Appendix B), and the $\Delta D_{i}$ 's and $\Delta R_{i j}$ 's link all the $\Delta S_{j}$ 's through $\mathbb{R}^{-1}$. The principal tool is the covariance matrix $\mathbb{C}(\Delta \mathbf{S})$ :

$$
\mathbb{C}(\Delta \mathbf{S})=\left(\begin{array}{ccc}
\sigma^{2}\left(\Delta S_{1}\right) & \ldots & \operatorname{cov}\left(\Delta S_{1}, \Delta S_{N}\right) \\
\vdots & \ddots & \vdots \\
\operatorname{cov}\left(\Delta S_{N}, \Delta S_{1}\right) & \cdots & \sigma^{2}\left(\Delta S_{N}\right)
\end{array}\right),
$$

where $\operatorname{cov}\left(\Delta S_{j}, \Delta S_{l}\right)$ is the covariance between the $j$ and $l$ components of $\Delta \mathbf{S}$ in Eq. (8). $\mathbb{C}(\Delta \mathbf{S})$ is a symmetric matrix with diagonal elements $\sigma^{2}\left(\Delta S_{j}\right)$. (An alternative approach is the Monte Carlo method [6].)

$\mathbb{C}(\Delta \mathbf{S})$ may be straightforwardly deduced from the corresponding covariance matrices, $\mathbb{C}(\Delta \mathbf{D})$ and $\mathbb{C}([\Delta \mathbb{R}] \mathbf{S})$, for $\Delta D_{i}$ and $\Delta R_{i j}$ because Eq. (8) is linear. One finds [Appendix B and Ref. [7]]

$$
\mathbb{C}(\Delta \mathbf{S})=\left(\mathbb{R}^{-1}\right)[\mathbb{C}(\Delta \mathbf{D})+\mathbb{C}([\Delta \mathbb{R}] \mathbf{S})]\left(\mathbb{R}^{-1}\right)^{\mathrm{T}},
$$

where $\left(\mathbb{R}^{-1}\right)^{\mathrm{T}}$ denotes the transpose of $\mathbb{R}^{-1}$. Terms like $\operatorname{cov}\left(\Delta D_{i}, \Delta R_{i j}\right)$ do not appear in Eq. (11) because the response-function calibrations and diagnostic shot data are assumed independent. $\mathbb{C}(\Delta \mathbf{S})$ then leads directly to the variance $\sigma^{2}\left(\Delta \mathcal{F}_{\text {unfold }}\right)$ in $\mathcal{F}_{\text {unfold }}$ via Eq. (9):

$$
\sigma^{2}\left(\Delta \mathcal{F}_{\text {unfold }}\right)=\Delta \mathbf{E} \cdot\{[\mathbb{C}(\Delta \mathbf{S})] \Delta \mathbf{E}\},
$$

where $\Delta \mathbf{E}$ again represents the fixed unfold bin widths $\Delta E_{j}$. In the following examples, error propagation proceeds by first deriving $\mathbb{C}(\Delta \mathbf{D})$ and $\mathbb{C}([\Delta \mathbb{R}] \mathbf{S})$ from error models for $\Delta D_{i}$ and $\Delta R_{i j}$, then constructing $\mathbb{C}(\Delta \mathbf{S})$ and the $\sigma^{2}\left(\Delta S_{j}\right)$ 's from Eq. (11), and lastly connecting $\sigma^{2}\left(\Delta \mathcal{F}_{\text {unfold }}\right)$ to $\mathbb{C}(\Delta \mathbf{S})$ via Eq. (12).

For later reference, it is convenient to list the components of Eqs. (11) and (12):

$$
\operatorname{cov}\left(\Delta S_{j}, \Delta S_{l}\right)=\sum_{i=1}^{N} \sum_{k=1}^{N}\left(\mathbb{R}^{-1}\right)_{j i} \operatorname{cov}\left(\Delta Y_{i}, \Delta Y_{k}\right)\left(\mathbb{R}^{-1}\right)_{l k},
$$

where $\Delta Y_{m}=\Delta D_{m}+([\Delta \mathbb{R}] \mathbf{S})_{m}$, and

$$
\sigma^{2}\left(\Delta \mathcal{F}_{\text {unfold }}\right)=\sum_{j=1}^{N} \sum_{l=1}^{N}\left(\Delta E_{j}\right) \operatorname{cov}\left(\Delta S_{j}, \Delta S_{l}\right)\left(\Delta E_{l}\right) .
$$

\section{Example: Random data fluctuations $\Delta D_{i}$ only}

We focus first on random data perturbations $\Delta D_{i}$ and ignore response-function uncertainties. The $\Delta D_{i}$ 's may represent low-level additive signal noise seen in [8] and Pt. 1: Fig. 3. For simplicity, we assume not only that $N\left(0, \sigma^{2}\left[\Delta D_{i}\right]\right)$ but that all the $\Delta D_{i}$ 's are statistically independent of one another: i.e.,

$$
\operatorname{cov}\left(\Delta D_{i}, \Delta D_{k}\right)=\sigma^{2}\left(\Delta D_{i}\right) \delta_{i k}=\zeta_{i}^{2} d_{i}^{2} \delta_{i k},
$$

where $\delta_{i k}$ is the Kronecker delta function. Here, $\zeta_{i}$ is the data noise-to-signal ratio (NSR) in the $i$ th data channel, $\sigma\left(\Delta D_{i}\right) d_{i}^{-1}=\zeta_{i}\left(d_{i} \neq 0\right)$. It follows that $\mathbb{C}(\Delta \mathbf{D})$ for this error model is diagonal.

Substituting $\mathbb{C}(\Delta \mathbf{D})$ into Eq. (11) and ignoring $\mathbb{C}([\Delta \mathbb{R}] \mathbf{S})$, one finds $\mathbb{C}(\Delta \mathbf{S})$. The $j$, lth element is 


$$
\mathbb{C}(\Delta \mathbf{S})_{j l}=\operatorname{cov}\left(\Delta S_{j}, \Delta S_{l}\right)=\sum_{i=1}^{N}\left(\mathbb{R}^{-1}\right)_{j i}\left(\mathbb{R}^{-1}\right)_{l i} d_{i}^{2} \zeta_{i}^{2}
$$

For the $Z$ diagnostic, $\mathbb{C}(\Delta \mathbf{S})$ is not diagonal due to the overlap of the $R_{i}(E)$ 's. Thus, while the data perturbations may be statistically independent, the $\Delta S_{j}$ 's are dependent.

The variances, $\sigma^{2}\left(\Delta S_{j}\right)$, and $\sigma^{2}\left(\Delta \mathcal{F}_{\text {unfold }}\right)$, for $\Delta S_{j}$ and $\Delta \mathcal{F}_{\text {unfold }}$, respectively, are then (Appendix C)

$$
\sigma^{2}\left(\Delta S_{j}\right)=\mathbb{C}(\Delta \mathbf{S})_{j j}=\sum_{i=1}^{N}\left(\mathbb{R}^{-1}\right)_{j i}^{2} d_{i}^{2} \zeta_{i}^{2},
$$

and, after some manipulation,

$$
\sigma^{2}\left(\Delta \mathcal{F}_{\text {unfold }}\right)=\sum_{i=1}^{N}\left[\sum_{j=1}^{N} \Delta E_{j}\left(\mathbb{R}^{-1}\right)_{j i}\right]^{2} d_{i}^{2} \zeta_{i}^{2} .
$$

To appreciate the propagation of data noise into flux uncertainties, it is useful to assume that the data NSR's are independent of channel and relatively small: i.e., $\sigma\left(\Delta D_{i}\right) d_{i}^{-1}=\zeta_{i}=\zeta \ll 1$. Then by factoring $\zeta^{2}$ out of Eqs. (17) and (18), one may define the $N+1$ parameters, $\nu_{j} \equiv \zeta^{-1} \sigma\left(\Delta S_{j}\right) S_{j}^{-1}$ and $\nu_{\mathcal{F}} \equiv \zeta^{-1} \sigma\left(\Delta \mathcal{F}_{\text {unfold }}\right) \mathcal{F}_{\text {unfold }}^{-1}$, which via Eqs. (4), (5), (17), and (18) reduce to

$$
\nu_{j} \equiv \frac{1}{\zeta} \frac{\sigma\left(\Delta S_{j}\right)}{S_{j}}=\frac{\sqrt{\sum_{i=1}^{N}\left(\mathbb{R}^{-1}\right)_{j i}^{2} d_{i}^{2}}}{\sum_{i=1}^{N}\left(\mathbb{R}^{-1}\right)_{j i} d_{i}}
$$

and

$$
\nu_{\mathcal{F}} \equiv \frac{1}{\zeta} \frac{\sigma\left(\Delta \mathcal{F}_{\text {unfold }}\right)}{\mathcal{F}_{\text {unfold }}}=\frac{\sqrt{\sum_{i=1}^{N}\left[\sum_{j=1}^{N} \Delta E_{j}\left(\mathbb{R}^{-1}\right)_{j i}\right]^{2} d_{i}^{2}}}{\sum_{i=1}^{N}\left[\sum_{j=1}^{N} \Delta E_{j}\left(\mathbb{R}^{-1}\right)_{j i}\right] d_{i}},
$$

where the noise-free x-ray $d_{i}$ has been substituted for $D_{i}$, since by assumption $\zeta \ll 1$. Such parameters are called noise factors (NF) [2] because each is the ratio of an "output" NSR [e.g., $\sigma\left(\Delta S_{j}\right) S_{j}^{-1}$ ] to an "input" NSR [e.g., ל]. A $\nu>1$ indicates amplification of input data noise (or uncertainty) in the unfold process; $\nu<1$ signifies noise reduction - typically, by averaging processes.

As defined, these NF's depend on the incident spectrum through $d_{i}$. Such dependences, $\nu_{j}[T]$ and $\nu_{\mathcal{F}}[T]$, are shown in Figs. 1(a) and 1(b) for the Planckian spectra $S_{b b}(E, T)$, studied previously (Pt. 1: Sec. IV). In Fig. 1(a), one sees strongly temperature-dependent amplifications $\nu_{j}[T]$ of relative data noise into the unfold $\operatorname{NSR}\left(S_{j}\right)$ for the peripheral unfold bins, an easily explained result: at the highest temperatures, $S_{1}$ in bin $\left[\Delta E_{1}\right]$ depends on small differences in similar data values due to response-function overlap, while at the lowest temperatures, $S_{5}$ in bin $\left[\Delta E_{5}\right]$ is constructed from essentially no signal [Eq. (2), Pt. 1: Eq. (25), and Pt. 1: Figs. 1 and 4]. In the central bins, $\nu_{2}[T], \nu_{3}[T] \approx 1$ because the responses $R_{2}(E)$ and $R_{3}(E)$ are more localized spectrally than the others and thus have fewer contributing data channels and correspondingly less data noise [Pt. 1: Fig. 1, and Pt. 1: Eq. (25)]. An upper bound to the $\nu_{j}$ 's is set by the cond $(\mathbb{R})$ [[9-14]; Pt. 1: Sec. IIIG], and one may verify that the inequality, $\left.\zeta^{-1}\left[\sum_{j=1}^{N} \sigma^{2}\left(\Delta S_{j}\right)\right]^{1 / 2}\left[\sum_{j=1}^{N} S_{j}^{2}\right)\right]^{-1 / 2} \leq \operatorname{cond}(\mathbb{R}) \cong 11$, in fact holds for these simulations. [Excluded from Fig. 1(a) are simulations in which some $S_{j}<0$, i.e., for $T<150 \mathrm{eV}$.]

By contrast, the flux NF $\nu_{\mathcal{F}}[T]$ in Fig. 1(b) shows a reduction in $\operatorname{NSR}\left(\mathcal{F}_{\text {unfold }}\right)$ relative to data noise and a gentle dependence on spectral shape. (All Planckian simulations are included because $\mathcal{F}_{\text {unfold }}>0$ in each.) This difference in behavior between $\nu_{\mathcal{F}}$ and $\nu_{j}$ occurs because $\mathcal{F}_{\text {unfold }}$ is an average over the $S_{j}$ 's (thus benefiting from the central limit theorem $[1,7,15])$, whereas the $S_{j}$ 's themselves depend on differences among the channel data $D_{i}$ [15]. Applying the Cauchy-Schwarz inequality [16-18] to Eq. (18), one derives formal bounds, $(N-n)^{-1 / 2} \lesssim \nu_{\mathcal{F}} \lesssim 1$, for the effective number of unfold channels $(N-n)$ that dominate datanoise propagation into $\mathcal{F}_{\text {unfold }}$; Fig. 1(b) suggests that 1-3 statistically independent channels characterize $\sigma^{2}\left(\Delta \mathcal{F}_{\text {unfold }}\right)$ for the spectra considered. Figure 1(b) also shows the corresponding flux noise factor for the flux estimate, $\mathcal{F}_{L S}$, based on the same response functions but without an unfolding procedure (cf. Ref. [8] and Pt. 1: Sec. V). The similarity in results for these independent measures lends support to our definition of $\mathcal{F}_{\text {unfold }}$ (cf. Sec. III C).

\section{Example: Calibrational errors only}

A second application of Eqs. (11) and (12) highlights random perturbations $\Delta R_{i}(E)$, which refer to errors in the responses $R_{i}$ due to uncertainty in the calibrational fit parameters (Pt. 1: Appendix B). Such perturbations enter Eqs. (8) and (9) as the elements $\Delta R_{i j}$ of $\Delta \mathbb{R}$ and propagate into $\sigma^{2}\left(\Delta S_{j}\right)$ and $\sigma^{2}\left(\Delta \mathcal{F}_{\text {unfold }}\right)$ : thus, $\operatorname{cov}\left(\Delta R_{i j}, \Delta R_{k l}\right) \rightarrow$ $\mathbb{C}([\Delta \mathbb{R}] \mathbf{S}) \rightarrow \mathbb{C}(\Delta \mathbf{S}) \rightarrow \sigma^{2}(\Delta \mathcal{F})$. As in the previous example, the analysis requires an error model, which here requires $N^{2}$ random variables, instead of $N$ for $\Delta D_{i}$ (cf. Appendix D). This model, nevertheless, yields similar expressions for $\sigma^{2}\left(\Delta S_{j}\right)$ and $\sigma^{2}\left(\Delta \mathcal{F}_{\text {unfold }}\right)$ as Eqs. (17) and (18).

Three assumptions define this error model. First, each channel $i$ of the filtered-XRD array consists of a known, single-component filter and a photocathode, calibrated separately. Thus, for $i=1, \ldots, N$

$$
R_{i}(E) \equiv A_{i} \mu_{x r d, i}(E) \exp \left[-\tau_{i} \mu_{i, f}(E)\right]
$$

where the x-ray coefficients, $\mu_{i, f}$ and $\mu_{x r d, i}$, for each filterXRD pair include known weight fractions [19] of atomic elements, compounds, or composites (cf. Appendix D). The terms, $A_{i}$ and $\tau_{i}$, are experimental fit parameters with associated errors, $\Delta A_{i}$ and $\Delta \tau_{i}$. Second, $\Delta A_{i}$ and 
$\Delta \tau_{i}$ derive from independent distributions, $N\left[0, \sigma^{2}\left(\Delta A_{i}\right)\right]$ and $N\left[0, \sigma^{2}\left(\Delta \tau_{i}\right)\right]$; hence, $\operatorname{cov}\left(\Delta A_{i}, \Delta \tau_{k}\right)=0$ for all $i, k$ and $\operatorname{cov}\left(\Delta A_{i}, \Delta A_{k}\right)=\operatorname{cov}\left(\Delta \tau_{i}, \Delta \tau_{k}\right)=0$ for $i \neq k$. Third, the NSR's of the fit constants, $\sigma\left(\Delta A_{i}\right) A_{i}^{-1} \equiv \alpha_{i}$ and $\sigma\left(\Delta \tau_{i}\right) \tau_{i}^{-1} \equiv \theta_{i}$, are assumed to be small $\left(\alpha_{i}, \theta_{i} \ll 1\right)$, so that again $d_{i} \cong D_{i}$.

The error-propagation process begins with definitions of the perturbations, $\Delta R_{i}(E)$ and $\Delta R_{i m}$, caused by uncertainty in the fit constants. To first order, the variation in Eq. (21) due to $\Delta A_{i}$ and $\Delta \tau_{i}$ is

$$
\Delta R_{i}(E)=\left[\frac{\Delta A_{i}}{A_{i}}\right] R_{i}(E)-\left[\frac{\Delta \tau_{i}}{\tau_{i}}\right]\left[\tau_{i} \mu_{i, f}(E)\right] R_{i}(E),
$$

and the corresponding perturbation $\Delta R_{i m}$ to the matrix element $R_{i m}$ is found by integrating Eq. (22) over each unfold bin $\left[\Delta E_{m}\right]$, as in Eq. (3):

$$
\Delta R_{\text {im }}=\left\{\frac{\Delta A_{i}}{A_{i}}-\frac{\Delta \tau_{i}}{\tau_{i}}\left\langle\tau_{i} \mu_{i, f}\right\rangle_{m}\right\} R_{i m}=(\Delta \mathbb{R})_{\mathrm{im}},
$$

where the unitless quantity, $\left\langle\tau_{i} \mu_{i, f}\right\rangle_{m} \equiv$ $\tau_{i} R_{\mathrm{im}}^{-1} \int_{E_{m}}^{E_{m+1}} R_{i}(E) \mu_{i, f}(E) d E$, is an average of the exponent of the filter transmission [Eq. (21)] over unfold bin $\left[\Delta E_{m}\right]$ and weighted by $R_{i}(E) / R_{i m}$.

When $\Delta A_{i}$ and $\Delta \tau_{i}$ are random numbers, this model statistically correlates intrachannel matrix elements, $\Delta R_{i m}$ with $\Delta R_{i n}$, because the same experimental fit parameters, $A_{i}$ and $\tau_{i}$, appear in the various bins, $m$ and $n$; however, interchannel elements, $\Delta R_{i m}$ and $\Delta R_{k n}(i \neq k)$, are uncorrelated due to our second model assumption. The statistical covariances, $\operatorname{cov}\left(\Delta R_{i j}, \Delta R_{k l}\right)$, between these elements of $\Delta \mathbb{R}$ are then straightforwardly derived (Appendix D) from Eq. (22), and are used to estimate $\mathbb{C}[(\Delta \mathbb{R}) \mathbf{S}]$, where $\mathbf{S}$ represents a nominal unfold estimate. One finds that $\mathbb{C}[(\Delta \mathbb{R}) \mathbf{S}]$ is diagonal, as was $\mathbb{C}(\Delta \mathbf{D})$ in the previous example [Eq. (15)].

Propagated estimates of $\mathbb{C}(\Delta S)$ and $\sigma^{2}\left(\Delta \mathcal{F}_{\text {unfold }}\right)$ can then be computed from Eqs. (11) and (12) (Appendix D). The propagated uncertainties of interest are

$$
\sigma^{2}\left(\Delta S_{j}\right)=\sum_{i=1}^{N}\left(\mathbb{R}^{-1}\right)_{j i}^{2} d_{i}^{2}\left(\alpha_{i}^{2}+\left\langle\left\langle\tau_{i} \mu_{i, f}\right\rangle\right\rangle^{2} \theta_{i}^{2}\right)
$$

and

$$
\begin{aligned}
\sigma^{2}\left(\Delta \mathcal{F}_{\text {unfold }}\right)= & \sum_{i=1}^{N}\left[\sum_{j=1}^{N} \Delta E_{j}\left(\mathbb{R}^{-1}\right)_{j i}\right]^{2} \\
& \times\left(\alpha_{i}^{2}+\left\langle\left\langle\tau_{i} \mu_{i, f}\right\rangle\right\rangle^{2} \theta_{i}^{2}\right) d_{i}^{2},
\end{aligned}
$$

where we have defined a spectrally dependent, double average, $\left\langle\left\langle\tau_{i} \mu_{i, f}\right\rangle\right\rangle \equiv \sum_{m=1}^{N} R_{i m} S_{m} d_{i}^{-1}\left\langle\tau_{i} \mu_{i, f}\right\rangle_{m}$. Equations (24) and (25) are identical to Eqs. (17) and (18), except that the data NSR's $\zeta_{i}$ are replaced by $\sqrt{\alpha_{i}^{2}+\left\langle\left\langle\tau_{i} \mu_{i, f}\right\rangle\right\rangle^{2} \theta_{i}^{2}}$. Thus, under this error model, the unfold algorithm produces similar spectral behavior (amplification or reduction) for calibrational uncertainties as for the data-noise model [Figs. 1(a) and 1(b)].

\section{Example: A first-order analysis of random errors for Z-shot 165}

Since shot-time noise and calibrational uncertainties for the $Z$ diagnostic are assumed statistically independent, their corresponding error models [Eq. (17), (18), (24), and (25)] may be combined in quadrature to estimate first-order uncertainties, $\sigma\left(\Delta S_{j}\right)$ and $\sigma\left(\Delta \mathcal{F}_{\text {unfold }}\right)$, in real experiments. Thus, one has

$$
\sigma^{2}\left(\Delta S_{j}\right)=\sum_{i=1}^{N}\left(\mathbb{R}^{-1}\right)_{j i}^{2} d_{i}^{2} \xi_{i}^{2}
$$

and

$$
\sigma^{2}\left(\Delta \mathcal{F}_{\text {unfold }}\right)=\sum_{i=1}^{N}\left(\sum_{j=1}^{N} \Delta E_{j}\left(\mathbb{R}^{-1}\right)_{j i}\right)^{2} d_{i}^{2} \xi_{i}^{2},
$$

where $\xi_{i}$, an effective input NSR, is defined by $\xi_{i}^{2}=$ $\sigma^{2}\left(\Delta D_{i}\right) d_{i}^{-2}+\sigma^{2}\left(\Delta A_{i}\right) A_{i}^{-2}+\left\langle\left\langle\tau_{i} \mu_{i, f}\right\rangle\right\rangle^{2} \sigma^{2}\left(\Delta \tau_{i}\right) \tau_{i}^{-2}$.

More realistic error models contribute additional terms (cf. Example 4, below, and Appendix D).

This analysis was applied to filtered XRD, channel data from $Z$-shot 165 . For this discussion, the calibrated response functions were $R_{1 b}(E), \ldots, R_{5 b}(E)$ (Pt. 1: Fig. 2), and the data values $D_{i} \approx d_{i}$ were taken at peak voltage (Pt. 1: Fig. 3). The nominal unfold coefficients $S_{j}$ were obtained from Eq. (2). Noise-to-signal ratios were then estimated as follows: $\sigma\left(\Delta D_{i}\right) D_{i}^{-1} \cong \sigma\left(\Delta D_{i}\right) d_{i}^{-1} \lesssim 10 \%$; $\sigma\left(\Delta A_{i}\right) A_{i}^{-1} \lesssim 10 \%-15 \%$ [4]; and $\sigma\left(\Delta \tau_{i}\right) \tau_{i}^{-1} \lesssim 5 \%$ [4]. The double average $\left\langle\left\langle\tau_{i} \mu_{i, f}\right\rangle\right\rangle$ varies from $\sim 1.5-2.5$ for Planckian spectra of temperatures, $150-250 \mathrm{eV}$, and is nearly independent of channel; we choose $\left\langle\left\langle\tau_{i} \mu_{i, f}\right\rangle\right\rangle=2$. Hence, a first-order estimate of the effective experimental NSR in Eqs. (26) and (27) is $\xi_{i}=$ $\sqrt{(0.1)^{2}+(0.125)^{2}+(2)^{2}(0.05)^{2}} \approx 0.2$; the corresponding experimental uncertainty, $\xi_{i} d_{i} \cong \xi D_{i}$, is then $\sim 20 \%$ of the measured data, of which calibrational uncertainties outweigh data noise at this sample time.

Figure 2(a) shows the unfolded (histogram) spectrum at peak emitted x-ray power with these error estimates. For clarity, the solid points centered on horizontal bars denote the nominal unfold values $S_{j}$ and the bin widths $\Delta E_{j}$, respectively, of $S_{\text {unfold }}(E)$. The vertical error bars appended to $S_{\text {unfold }}(E)$ represent $S_{j} \pm \sigma\left(\Delta S_{j}\right)$. In general agreement with Fig. 1(a), the peripheral unfold bins show the largest binwise NSR's. The estimated flux for this peak-power spectrum is $\mathcal{F}_{\text {unfold }} \pm \sigma\left(\Delta \mathcal{F}_{\text {unfold }}\right)=[6.6 \pm 0.8] \times$ $10^{12} \mathrm{~W} \mathrm{sr}^{-1}$; the NSR of $\mathcal{F}_{\text {unfold }}$ is then $\sim 12 \%$, which is a reduction of the input NSR (20\%) by a factor of $\sim 0.6$, in general agreement with Fig. 1(b). 

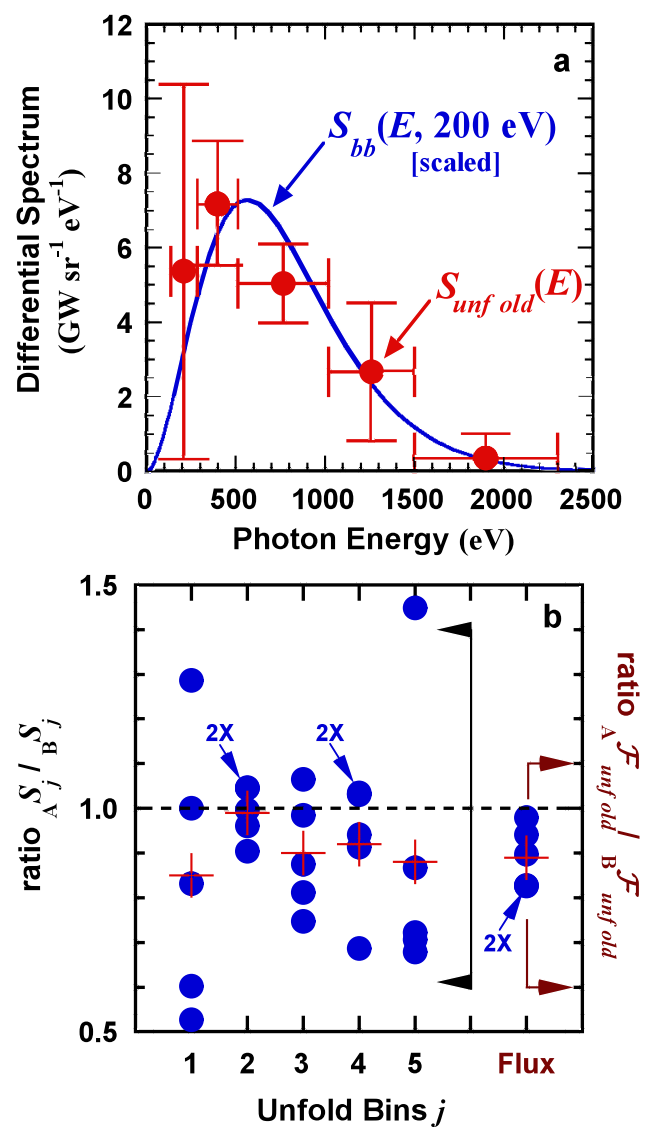

FIG. 2. Estimated experimental uncertainties for the $Z$ diagnostic, and fluctuations for repeated experiments. Part (a) shows the unfolded peak-power spectrum for $Z$-shot 165 (cf. Pt. 1: Figs. 3 and 4$)$. Here, for clarity, $S_{\text {unfold }}\left(E\right.$, $\left.t_{\text {peak }}\right)$ is denoted by solid (red) points at the midpoint of each unfold bin (Pt. 1: Table II), with horizontal bars indicating the bin widths; the ordinates $\left[\mathrm{GW} \mathrm{sr}^{-1} \mathrm{eV}^{-1}\right]$ are the unfold coefficients, $S_{j}$. Vertical error bars give the combined, propagated uncertainties, $\pm \sigma\left(\Delta S_{j}\right)$. The blue curve is a $200-\mathrm{eV}$ Planckian spectrum [GW sr ${ }^{-1} \mathrm{eV}^{-1} \mathrm{~cm}^{-2}$ ] scaled by integrated flux to the same units. Part (b) compares unfold coefficients and flux for two independent, filtered-XRD arrays (denoted "A" and "B"), which were simultaneously fielded on five, nominally identical, tungsten $z$-pinch shots at the $Z$ facility (Z-shots $163-167$ ). The solid points are the binwise unfold ratios, ${ }_{\mathrm{A}} S_{j} /{ }_{\mathrm{B}} S_{j}$, for each shot at peak power (left scale); the additional points at the right side of the figure are the corresponding unfold flux ratios, ${ }_{\mathrm{A}} \mathcal{F}_{\text {unfold }} / \mathcal{F}_{\text {unfold }}$ (right axis, same scale). The notation " $2 \mathrm{X}$ " indicates two nearly identical ratios; and the red "+" symbols denote the sample ratio mean for each bin $j$ and the flux estimate.

Because this spectral unfold includes both data and calibrational uncertainties, it is appropriate to compare the resulting spectrum to theoretical expectations. One such hypothesis, already entertained (Pt. 1: Sec. II D), is that the incident spectrum is nearly Planckian, $S\left(E, t_{\text {peak }}\right) \cong S_{b b}(E, T \approx 200 \mathrm{eV})$. Figure 2(a) includes this spectrum, scaled for comparison with $S_{\text {unfold }}(E)$. Specifically, the continuous blue line in the figure is
$S_{b b}(E$, scaled $)=\left(\mathcal{F}_{\text {unfold }} \mathcal{F}_{[\Delta E]}^{-1}\right) S_{b b}(E, 200 \mathrm{eV}), \quad$ where $\mathcal{F}_{[\Delta E]}$ is calculated from $S_{b b}$ over the same interval $[\Delta E]$ as $\mathcal{F}_{\text {unfold }}$. [Typical units for $S_{\text {unfold }}$ and $S_{b b}$ differ by source area, which the $Z$ diagnostic does not measure, so this scaling is equivalent to comparing the flux-normalized shapes, $\underline{S}_{\text {unfold }}$ and $\underline{S}_{b b}$ (Pt. 2: Table I; Pt. 1: Sec. III A)]. One sees in the figure that $S_{\text {unfold }}(E)$ is similar to $S_{b b}(E$, scaled $)$-especially in the high-energy tail-but appears to peak at a somewhat lower x-ray energy; hence, $S_{\text {unfold }}(E)$ may have a lower "color temperature" than $S_{b b}(E, 200 \mathrm{eV})$. The indicated uncertainties in $S_{\text {unfold }}(E)$, however, make this inference speculative.

A more stable appraisal of spectral or "radiation" temperature for $S_{\text {unfold }}$ is based directly on $\mathcal{F}_{\text {unfold }}$. This assessment, however, requires additional experimental information: in particular, one needs an emission model for the x-ray-emitting plasma source and a cross-sectional area. Assuming, first, that the z-pinch source in Z-shot 165 was uniform and Lambertian [2,20,21], one infers from $\mathcal{F}_{\text {unfold }}$ a peak, emitted x-ray power of $190 \mathrm{TW} \pm 12 \%$ into $4 \pi$ sr (Pt. 1: Appendix A). Second, x-ray framing-camera photographs in this shot show the $z$ pinch at stagnation as roughly cylindrical ( $2 \mathrm{~cm}$ high and $\sim 2 \mathrm{~mm}$ in diameter), albeit with many hot spots. A blackbody of these dimensions and radiating $190 \mathrm{TW}$ has a temperature of $\sim 196 \mathrm{eV}$ [20-22], in reasonable agreement with the hypothesized spectrum $S_{b b}(E, 200 \mathrm{eV})$.

\section{Example: Comparisons of paired filtered-XRD arrays}

The validity of these error models for the $Z$ diagnostic can be tested by comparing spectral unfolds and flux estimates from two such diagnostics fielded together on a sequence of x-ray shots. Since the source may itself vary from shot to shot, we modify the data model by replacing $S(E)$ at fixed time in Eq. (1) by $S(E)+\Delta S(E)$, where $\Delta S(E)$ represents a random spectral variation, $N\left[0, \sigma^{2}(S)\right]$. Then the recorded data in the $i$ th channel of diagnostic $X$ have the following form:

$$
\begin{aligned}
{ }_{X} D_{i}= & \int_{0}^{E_{\mathrm{MAX}}}\left[{ }_{X} R_{i}(E)+{ }_{X} \Delta R_{i}(E)\right] S(E) d E \\
& +\left\{\int_{0}^{E_{\mathrm{MAX}}}\left[{ }_{X} R_{i}(E)+{ }_{X} \Delta R_{i}(E)\right] \Delta S(E) d E\right. \\
& \left.+{ }_{X} \Delta D_{i}\right\},
\end{aligned}
$$

where ${ }_{X} R_{i}(E)$ is the calibrated response function with error ${ }_{X} \Delta R_{i}(E)$, and ${ }_{X} \Delta D_{i}$ is signal noise. If the net responses, ${ }_{X} R_{i}(E)+{ }_{X} \Delta R_{i}(E)$, are constant for all the shots, then the first term on the right of Eq. (28) varies by channel, but not by shot: it carries calibrational bias into the unfold. The second term (in braces) in Eq. (28), on the other hand, encodes shot-to-shot variations about the calibrational bias and data noise. 
Two independent filtered-XRD arrays, as described for the $Z$-shot 165 (Sec. II C 3), were simultaneously fielded on five consecutive tungsten $z$-pinch shots ( $Z$-shots 163-167) over the course of $\sim 1$ week. (Calibrational drift was thus assumed negligible over this time period.) The two diagnostics (labeled, $X=$ "A" and "B") were located at distances 24 and $19 \mathrm{~m}$, respectively, from the $z$-pinch $\mathrm{x}$-ray source, which was replaced after each shot. For this sequence of experiments the configurations of the wire array, the $Z$ accelerator, and the filtered-XRD diagnostics were held fixed, so that only relatively small spectral variations were expected. Channel data from each diagnostic were unfolded via Eq. (2), using the appropriate inverse matrices $\left({ }_{A} \mathbb{R}^{-1}\right.$ and $\left.{ }_{B} \mathbb{R}^{-1}\right)$, which were derived from up-to-date calibrations and the same energy bins. Time-varying, unfold coefficients $\left({ }_{\mathrm{A}} S_{j}\right.$ and $\left.{ }_{\mathrm{B}} S_{j}\right)$ and fluxes $\left(\mathcal{F}_{\text {unfold }} \mathrm{A}\right.$ and ${ }_{\mathrm{B}} \mathcal{F}_{\text {unfold }}$ ) were obtained for each shot.

Figure 2(b) compares the unfolded results at $t_{\text {peak }}$ in this shot sequence (solid points). Binwise coefficient and flux ratios, $\left({ }_{\mathrm{A}} S_{j}\right)\left({ }_{\mathrm{B}} S_{j}\right)^{-1} \quad(j=1, \ldots, 5)$ and $\left({ }_{\mathrm{A}} \mathcal{F}_{\text {unfold }}\right) \times$ $\left({ }_{\mathrm{B}} \mathcal{F}_{\text {unfold }}\right)^{-1}$, are shown. One sees that the five-shot average (red crosses) of $\left({ }_{\mathrm{A}} S_{j}\right)\left({ }_{\mathrm{B}} S_{j}\right)^{-1}$ differs from 1 by $\lesssim 15 \%$, and the corresponding mean of $\left({ }_{\mathrm{A}} \mathcal{F}_{\text {unfold }}\right)\left({ }_{\mathrm{B}} \mathcal{F}_{\text {unfold }}\right)^{-1}$ differs from 1 by $11 \%$.

These results are consistent with the model in Eq. (28), which combines the data and calibrational error models developed in previous examples of this section. For example, by simulations [Pt. 1: Sec. IVA, Fig. 10(a)] and theoretical arguments [23], one does expect these ratios to be close to unity. Moreover, the average values in Fig. 2(b) suggest first-order bias effects ${ }_{X} \Delta R_{i}(E)$ of $\sim 10 \%$, probably traceable to the calibrations themselves and to other fixed experimental parameters. Lastly, the shot-to-shot channel-wise fluctuations in $\left({ }_{\mathrm{A}} S_{j}\right)\left({ }_{\mathrm{B}} S_{j}\right)^{-1}$ and $\left({ }_{\mathrm{A}} \mathcal{F}_{\text {unfold }}\right) \times$ $\left({ }_{\mathrm{B}} \mathcal{F}_{\text {unfold }}\right)^{-1}$ are qualitatively similar to the noise factors in Figs. 1(a) and 1(b) for $T=200 \mathrm{eV}$ and would be compatible with shot-dependent terms, ${ }_{x} \Delta D_{i}$ and $\Delta S(E)$.

\section{GENERALIZATION OF THE UNFOLD PROCEDURE}

To this point, our testing of the histogram unfold algorithm with simulations has relied heavily on Planckian spectra. However, a more abstract approach is needed to make sense of the algorithm's deterministic behavior for arbitrary spectra. The key to this analysis is the functional separability [24] of the detection/unfold process. It allows this operation to be reduced to the integrated product of a postulated spectral function with response-based "passband" functions, without explicit data computations (cf. Pt. 1: Fig. 4). The goal of this approach is to understand quantitatively (a) the distortions observed in part 1 (Sec. IV), (b) how violations of the diagnostic model (Pt. 1: Table I) are processed, (c) why negative unfold values appear, and (d) how such artifacts should be treated in a flux estimate. This analysis does not supersede the principal unfold equations [Eqs. (2)-(5)], which address experimental data.

\section{A. An abstract view of the unfold problem}

The unfold problem of Eq. (1) can be posed mathematically in terms of four interconnected abstract structures, within which previous sections of this article may be interpreted. These structures include: (a) the Hilbert space $\mathrm{L}_{2}\left[0, E_{\mathrm{MAX}}\right]$ of real functions $f(E)$ defined over $\left[0, E_{\mathrm{MAX}}\right]$; (b) a linear space $\mathrm{D}$ of vectors $\mathbf{D}$; (c) a forward linear mapping $\mathcal{M}: \mathrm{L}_{2} \Rightarrow \mathrm{D}$; and (d) an inverse linear mapping, $\mathcal{M}_{\mathrm{BD}}^{-1}: \mathrm{D} \Rightarrow \mathrm{B}$, where $\mathrm{B}$ is proper subset of $\mathrm{L}_{2}$.

The properties of such abstract structures are, of course, well known, but it is useful to highlight here certain properties of each before applying them to the unfold algorithm (cf. Table I).

The real Hilbert space $\mathrm{L}_{2}\left[0, E_{\mathrm{MAX}}\right]$ is an infinitedimensional set $[10,16,17,25-34]$ of real functions $f(E)$ that are square integrable $\left[(f \mid f)=\int_{0}^{E_{\mathrm{MAX}}} f^{2}(E) d E<\infty\right]$ in the interval $\left[0, E_{\mathrm{MAX}}\right]$. (The Dirac delta function $[2,24,35]$ is excluded.) $L_{2}$ includes functions of physical relevance to spectral emission from a $z$ pinch and to the $Z$ diagnostic: e.g., broad continuum spectra $S(E)$ (Pt. 1: Table I); the $N$ response functions $R_{i}$ (Pt. 1: Fig. 2); and the $N$ histogram basis functions $B_{j}$ [Pt. 1, Eq. (13)]. Other functions in $\mathrm{L}_{2}$ need have no physical relevance and can, in fact, be troublesome for unfolding: e.g., null functions $f_{0}$ have the property, $\left(f_{0} \mid R_{i}\right) \equiv \int_{0}^{E_{\mathrm{MAX}}} f_{0}(E) R_{i}(E) d E \equiv 0$ for all the $R_{i}$ 's, and form their own infinite-dimensional subspace $\mathrm{N}$ in $\mathrm{L}_{2}$ (Pt. 1: Sec. IIC). $\mathrm{L}_{2}$ also contains a closed $N$-dimensional subspace B made of all linear combinations of the histogram basis functions, $\sum_{j=1}^{N} f_{j} B_{j}(E)$.

The vector space $\mathrm{D}$ comprises all $N$-dimensional real vectors of the form $\mathbf{D}=\left(D_{1}, \ldots, D_{N}\right)$ with components $D_{i}$. The inner product $\mathbf{D} \cdot \mathbf{D}^{\prime}$ of two vectors, $\mathbf{D}$ and $\mathbf{D}^{\prime}$, is defined as $\sum_{i=1}^{N} D_{i} D_{i}^{\prime}$, which leads to the Euclidian norm, $\|\mathbf{D}\| \equiv \sqrt{\mathbf{D} \cdot \mathbf{D}}$. Some vectors $\mathbf{D}$ represent experimental channel data in the $Z$ diagnostic.

A mapping $\mathcal{M}$ from $L_{2}$ to $D$ associates each function $f(E)$ in $\mathrm{L}_{2}$ with one (and only one) vector in $\mathrm{D}$. In the absence of perturbations $[\varepsilon=0]$, Eq. (1) defines such a mapping, linking $f(E)$ with the vector of $N$ inner products: that is, $\mathcal{M} f=\left[\left(R_{1} \mid f\right), \ldots,\left(R_{N} \mid f\right)\right]$, which describes x-ray detection in the context of the $Z$ diagnostic. This mapping is both linear (i.e., $\mathcal{M}\left[\alpha f_{1}+\beta f_{2}\right]=\alpha \mathcal{M} f_{1}+\beta \mathcal{M} f_{2}$ for functions $f_{1}, f_{2}$ and scalars $\alpha, \beta$ ) and continuous. As defined, $\mathcal{M}$ is perforce a many-to-one mapping because the dimension of $L_{2}$ exceeds the dimension of $D$. [In particular, the null functions $f_{0}(E)$ map into $\mathbf{0}=(0,0, \ldots, 0)$; cf. Pt. 1: Sec. IIC.] For the particular responses and basis functions here, this mapping has two additional properties: (a) each vector $\mathbf{D}$ in $\mathbf{D}$ is linked by $\mathcal{M}$ to at least some function $f(E)$ in $\mathrm{L}_{2}$; and (b) each vector D is associated by $\mathcal{M}$ with a unique member, 
$\sum_{j=1}^{N} f_{j} B_{j}(E)$, in subspace B $[10,28]$. (These properties obtain because the $R_{i}$ 's are linearly independent; and the $B_{j}$ 's are likewise independent and nonorthogonal to the $R_{i}$ 's; cf. Pt. 1: Sec. III.)

$\mathcal{M}$ may thus be exploited to define a formally separate mapping, $\mathcal{M}_{\mathrm{BD}}$ : $\mathrm{B} \Rightarrow \mathrm{D}$, between the $N$-dimensional spaces $B$ of $L_{2}$ and $D$. Simply, we define $\mathcal{M}_{\mathrm{BD}}$ to coincide with $\mathcal{M}$ over all the elements, $f_{B}=\sum_{j=1}^{N} f_{j} B_{j}(E)$, in $\mathrm{B}$; that is, $\mathcal{M}_{\mathrm{BD}}\left[f_{B}\right] \equiv \mathcal{M}\left[f_{B}\right] \equiv\left[\sum_{j=1}^{N} f_{j}\left(R_{1} \mid B_{j}\right), \ldots\right.$, $\left.\sum_{j=1}^{N} f_{j}\left(R_{N} \mid B_{j}\right)\right]$, which is a vector in $\mathrm{D}$. The reason for this secondary mapping will become clear presently. According to the particular properties, (a) and (b), of $\mathcal{M}$ above, $\mathcal{M}_{\mathrm{BD}}$ must be a one-to-one mapping. It must, therefore, have an inverse mapping, $\mathcal{M}_{\mathrm{BD}}^{-1}$ : $\mathrm{D} \Rightarrow \mathrm{B}$, from $\mathrm{D}$ onto $\mathrm{B}$, which can be shown to be both linear and continuous over D. In fact, the numerical process, $\left(f_{1}, \ldots, f_{N}\right)=$ $\mathbb{R}^{-1}\left(D_{1}, \ldots, D_{N}\right)$ defined in Eq. (4), just represents $\mathcal{M}_{\mathrm{BD}}^{-1} \mathbf{D}$, which describes the reconstruction for the histogram unfold algorithm.

It follows that, given arbitrary $f(E)$ in $\mathrm{L}_{2}$, it is possible to find the vector image, $\mathcal{M} f=\left(d_{1}, \ldots, d_{N}\right)$, in $\mathrm{D}$ and then to connect this vector uniquely to a histogram, $\sum_{j=1}^{N} f_{j} B_{j}(E)=\mathcal{M}_{\mathrm{BD}}^{-1}\left(d_{1}, \ldots, d_{N}\right)$ in $\mathrm{B} \subset \mathrm{L}_{2}$. This combined process, $\mathcal{U}_{f} \equiv \mathcal{M}_{\mathrm{BD}}^{-1}[\mathcal{M} f]$ or $\mathcal{M}_{\mathrm{BD}}^{-1} \mathcal{M} f$, we previously called collocation (Pt. 1, Sec. III E) and used it to test the unfold algorithm (Pt. 1: Sec. IV). That is, we applied the two parts of $\mathcal{U}$ in sequence to a prescribed spectrum $S(E)$ to obtain the histogram reconstruction, $S_{\text {unfold }}(E)$ [Pt. 2: Eq. (2)]. $\mathcal{M}$ and $\mathcal{M}_{\mathrm{BD}}^{-1}$ may be regarded as a transform/inverse-transform pair, in analogy to the Fourier transform and its inverse.

More formally, the composite mapping $\mathcal{U}$ here defines a continuous linear operator in $L_{2}$, i.e., a mapping of $L_{2}$ into itself, that allows one conveniently to compute the unfold algorithm for all spectral functions $f$ in $L_{2}$ without the intermediate step of explicitly simulating data (in contrast to Pt. 1: Sec. IV). $U$ then represents processes of detection and reconstruction in which pointwise detail and even some average information about $f$ is lost. The following subsection explores a representation of $\mathcal{U}$ that permits $\mathcal{U} f$ to be quickly visualized.

\section{B. Passband functions for the unfold coefficients \\ 1. Definition and properties}

According to the Riesz representation theorem [17,24], the image $\mathcal{K} f$ of a continuous linear operator $\mathcal{K}$ in $\mathrm{L}_{2}$ can always be written as an inner product of $f$ with a unique function $K\left(E, E^{\prime}\right)$ :

$$
\mathcal{K} f \equiv f_{\mathcal{K}}(E) \equiv \int_{0}^{E_{\mathrm{MAX}}} K\left(E, E^{\prime}\right) f\left(E^{\prime}\right) d E^{\prime},
$$

where $K\left(E, E^{\prime}\right)$ is bounded over the two-dimensional domain, $\left[0, E_{\mathrm{MAX}}\right] \times\left[0, E_{\mathrm{MAX}}\right], E$ is a fixed parameter with respect to the integral $[17,24,36]$, and $f_{\mathcal{K}}(E)$ is a function in $\mathrm{L}_{2}$. ( $K$ is sometimes called an averaging kernel, cf. [37] and Pt. 1: Sec. III C.) Hence, when $K\left(E, E^{\prime}\right)$ is known, the computation of $f_{\mathcal{K}}(E)$ is reduced to quadrature. The goal of this subsection is a similar description of $\mathcal{U}$, the simulation/unfold operator above [Sec. III A], which leads to a deeper understanding of how the unfold algorithm responds to arbitrary $f$.

To illustrate how Eq. (29) works, consider the linear operator $\mathcal{A}$ that associates $f(E)$ with the piecewiseconstant function, $\mathcal{A} f=f_{\mathrm{AVE}}(E)=\sum_{j=1}^{N}\langle f\rangle_{j} B_{j}(E)$, where the $\langle f\rangle_{j}$ 's are simple averages [cf. Eq. (6)] over the unfold bins $\left[\Delta E_{j}\right]$ (Pt. 1: Table II). Results of this operator were illustrated in part 1 of this article for Planckian spectra $S_{b b}(E, T)$ (Pt. 1: Fig. 8). An integral representation of $\mathcal{A}$ is obtained by substituting $A\left(E, E^{\prime}\right) \equiv$ $\sum_{j=1}^{N}\left(\Delta E_{j}\right)^{-1} B_{j}(E) B_{j}\left(E^{\prime}\right)$ for $K\left(E, E^{\prime}\right)$ in Eq. (29). To prove that this particular representation duplicates $\mathcal{A} f$, one notes that $E$ and $E^{\prime}$ are separable [24] in $A\left(E, E^{\prime}\right)$ so that

$$
\begin{aligned}
\int_{0}^{E_{\mathrm{MAX}}} A\left(E, E^{\prime}\right) f\left(E^{\prime}\right) d E^{\prime} \\
=\int_{0}^{E_{\mathrm{MAX}}}\left[\sum_{j=1}^{N}\left(\Delta E_{j}\right)^{-1} B_{j}(E) B_{j}\left(E^{\prime}\right)\right] f\left(E^{\prime}\right) d E^{\prime} \\
=\sum_{j=1}^{N}\left(\rho_{A, j} \mid f\right) B_{j}(E)=f_{\mathrm{AVE}}(E)=\mathcal{A} f,
\end{aligned}
$$

where

$$
\rho_{A, j}(E)=\frac{B_{j}(E)}{\Delta E_{j}} .
$$

We call $\rho_{A, j}$ an averaging passband function of $\mathcal{A}$ because the calculation of the coefficient $\langle f\rangle_{j}$ in bin $\left[\Delta E_{j}\right]$ is a simple integral: $\langle f\rangle_{j}=\left(\rho_{A, j} \mid f\right)=\int_{0}^{E_{\mathrm{MAX}}} \rho_{A, j}\left(E^{\prime}\right)$ $f\left(E^{\prime}\right) d E^{\prime}$. Thus, each $\rho_{A, j}$ plays the role of a sensitivity or filter function used in signal analysis [2,7,38-41], and $\left\{\rho_{A, j}\right\}_{j=1}^{N}$ provides everything one needs to know about the operation of $\mathcal{A}$. Figure 3(a) plots these passband functions. (The vertical axis of each $\rho_{A, j}$ is displaced for clarity.)

The passband concept similarly applies to the unfold operator, $\boldsymbol{U}=\mathcal{M}_{\mathrm{BD}}^{-1} \mathcal{M}$, which associates each $f(E)$ in $\mathrm{L}_{2}$ with a simulation/unfold reconstruction, $f_{\text {unfold }}(E)$. In this case, substituting the separable kernel [24] $U\left(E, E^{\prime}\right) \equiv$ $\sum_{j=1}^{N} \sum_{i=1}^{N}\left(\mathbb{R}^{-1}\right)_{j i} B_{j}(E) R_{i}\left(E^{\prime}\right)$ for $K\left(E, E^{\prime}\right)$ in Eq. (29) yields the representation

$$
\mathcal{U} f=\sum_{j=1}^{N}\left(\rho_{U, j} \mid f\right) B_{j}(E)=f_{\text {unfold }}(E),
$$

where the function

$$
\rho_{U, j}(E) \equiv \sum_{i=1}^{N}\left(\mathbb{R}^{-1}\right)_{j i} R_{i}(E)
$$



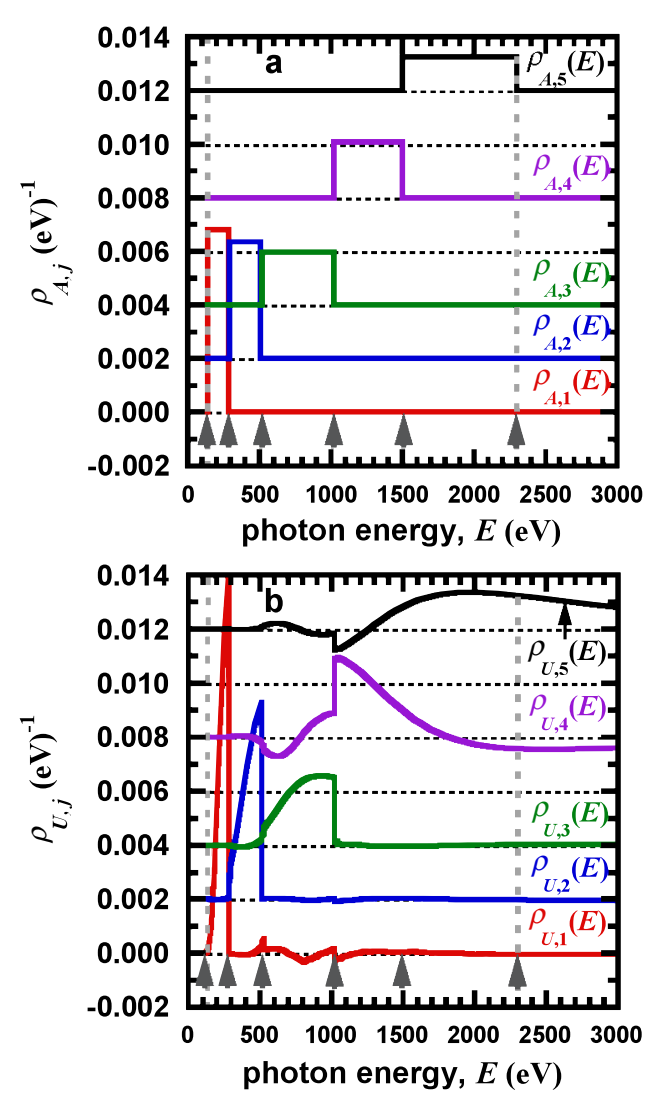

FIG. 3. Binwise passband functions, $\rho_{A, j}(E)$ and $\rho_{U, j}(E)$, for the averaging operator $\mathcal{A}$ and the unfold operator $\mathcal{U}=\mathcal{M}_{\mathrm{BD}}^{-1} \mathcal{M}$, respectively. In both figures, the baselines are displaced for clarity, dashed vertical lines denote the unfold interval $[\Delta E]$, and arrowheads indicate the unfold bin boundaries. Part (a) graphs averaging passband functions $\rho_{A, j}(E)$, each of constant value $\left(\Delta E_{j}\right)^{-1}$ within the $j$ th unfold bin, but zero elsewhere (Pt. 1: Table II). Part (b) shows the corresponding unfold passband functions $\rho_{U, j}(E)$, which exhibit x-ray absorptionedge features in the responses (Pt. 1: Fig. 1) but not the highenergy humps of $R_{1}, R_{2}, R_{3}$ (Pt. 1: Figs. 1 and 2). The $\rho_{U, j}(E)$ 's can be nonzero, and even negative valued, outside bin $\left[\Delta E_{j}\right]$.

is called an unfold passband function $\rho_{U, j}(E)$, analogous to $\rho_{A, j}(E)$ above, and where $\mathbb{R}^{-1}$ is the unfold matrix [cf. Eq. (3)]. Equation (33) defines each $\rho_{U, j}(E)$ as a linear combination of the response functions; and the terms, $\left(\rho_{U, j} \mid f\right)$, in Eq. (32) are the unfold coefficients (Pt. 1: Sec. IV $), f_{j}=\int_{0}^{E_{\mathrm{MAX}}} \rho_{U, j}\left(E^{\prime}\right) f\left(E^{\prime}\right) d E^{\prime}$, obtained here without explicit data calculations [Ref. [42], Eq. (1), and Pt. 1: Fig. 4]. Thus, each $\rho_{U, j}(E)$ is a spectral sensitivity for computing $f_{j}$ with arbitrary $f(E)$, and together these functions embed all the a priori conditions and behavior of the unfold algorithm. Figure 3(b) plots the $\rho_{U, j}(E)$ 's for responses, $R_{1}, \ldots, R_{5}$ (Pt. 1: Fig. 2).

It is instructive to compare the shapes and integral properties of $\rho_{A, j}(E)$ and $\rho_{U, j}(E)$. Defined solely by
$B_{j}(E)$, each averaging passband function $\rho_{A, j}(E)$ in Fig. 3(a) is positive and nonzero only in the $j$ th unfold bin. It follows that $\int_{E_{k}}^{E_{k+1}} \rho_{A, j}(E) d E=\delta_{j k}$ and $\left\langle\rho_{A, j}\right\rangle_{k}=$ $\left(\Delta E_{j}\right)^{-1} \delta_{j k}$ over arbitrary $\left[\Delta E_{k}\right]$. In addition, according to Eq. (30), the integral, $\int_{0}^{E_{\mathrm{MAX}}} \rho_{A, j}(E) f(E) d E$, returns $\langle f\rangle_{j}$, which is a "bin-localized" value for $f$. Both these properties recall the action of a Dirac delta function, but on a much cruder scale.

The unfold passband functions $\rho_{U, j}(E)$ in Fig. 3(b) show similar properties. Although composed of linear combinations of the $R_{i}(E)$ 's, the $\rho_{U, j}$ 's also exhibit nonzero behavior largely within the corresponding unfold bins $j-$ especially for $\rho_{U, 1}(E), \ldots, \rho_{U, 3}(E)$. The sharp peaks in these three passband functions are due to the characteristic x-ray absorption edges of $R_{1}(E), \ldots, R_{3}(E)$; but, the higher-energy "humps" visible in the response functions are absent in $\rho_{U, 1}(E), \ldots, \rho_{U, 3}(E)$. The higher-order passbands, $\rho_{U, 4}(E)$ and $\rho_{U, 5}(E)$, are broader than the comparable averaging passbands: in particular, both show noticeable spectral sensitivity above the upper cutoff of the unfold algorithm $\left(E_{\mathrm{HI}}=2300 \mathrm{eV}\right)$. They also display some negative behavior, which is absent in the $\rho_{A, j}$ 's. One can, nevertheless, show [43] that the same integral properties as above also apply to the $\rho_{U, j}$ 's: i.e., $\int_{E_{k}}^{E_{k+1}} \rho_{U, j}(E) d E=\delta_{j k}$, and $\left\langle\rho_{U, j}\right\rangle_{k}=\left(\Delta E_{j}\right)^{-1} \delta_{j k}$, plus the crude binwise localization value, $f_{j}$ [Eq. (32)].

\section{Applications of unfold passband functions}

Some previous assertions about how the unfold algorithm works can now be verified. We examine specifically: (a) Why do the unfolded and averaged spectra in Pt. 1: Figs. 7 and 8 agree as well as they do? (b) How does the unfold algorithm respond to spectra that violate assumptions in Pt. 1: Table I? And (c) what is the cause and impact of negative unfold coefficients [Pt. 1: Table III and Pt. 1: Figs. 8(c) and 10(b)]?

Agreement of $f_{\text {unfold }}(E)$ and $f_{\mathrm{AVE}}(E)$. - The first issue is addressed by generalizing the concept of absolute channelwise distortion between $f_{\text {unfold }}(E)$ and $f_{\mathrm{AVE}}(E)$ (Pt. 1: Sec. IV and Pt. 1: Figs. 7 and 8). We begin by isolating the part $\Delta f_{B}(E)$ of $f(E)$ that cannot be approximated by the set of basis functions $\left\{B_{k}(E)\right\}_{k=1}^{N}$ in B:

$$
\Delta f_{B}(E) \equiv f(E)-f_{\mathrm{AVE}}(E)=f(E)-\sum_{k=1}^{N}\langle f\rangle_{k} B_{k}(E)
$$

In a least-squares sense, $\sum_{k=1}^{N}\langle f\rangle_{k} B_{k}(E)$ represents the best unfold-histogram [44] approximation to $f(E)$. (In Pt. 1: Sec. IV, the norm $\left\|\Delta f_{B}\right\|$ described an rms measure of approximation for Pt. 1: Figs. 7 and 8.) It follows that because $\left(\Delta f_{B} \mid B_{k}\right) \equiv 0$ [Eq. (34)] for all the $B_{k}$ 's, $\Delta f_{\mathrm{B}}(E)$ must be either orthogonal to $B$ (i.e., reside outside $B$ ) or zero-valued (except at a finite number of points). 
We previously defined absolute unfold distortion by comparing the differences, $S_{j}-\langle S\rangle_{j}$, for prescribed spectra (Pt. 1: Sec. IV). This concept may be generalized as the operational difference, $\mathcal{U} f-\mathcal{A} f$, where

$$
\begin{aligned}
\mathcal{U} f-\mathcal{A} f & =f_{\text {unfold }}(E)-f_{\mathrm{AVE}}(E) \\
& \equiv \sum_{J=1}^{N}\left(\rho_{U, j}-\rho_{A, j} \mid f\right) B_{j}(E)
\end{aligned}
$$

[Eqs. (30) and (32)]. It follows [45] that

$$
f_{j}-\langle f\rangle_{j}=\int_{0}^{E_{\mathrm{MAX}}} \rho_{U, j}(E) \Delta f_{\mathrm{B}}(E) d E,
$$

which applies for arbitrary spectral functions. This result quantifies the inference (Pt. 1: Sec. IV) that binwise distortion of the unfold algorithm depends on (a) the nonconstant portion $\Delta f_{B}$ of $f(E)$ in each bin $\left[\Delta E_{j}\right]$ and (b) the response functions [Eq. (33)]. The observed binwise distortions in Pt. 1: Fig. 8 are now understandable. For incident spectra well approximated by $\sum_{k=1}^{N}\langle f\rangle_{k} B_{k}(E)$, $\Delta f_{\mathrm{B}}(E) \approx 0$ and thus $f_{j} \cong\langle f\rangle_{j}$ [cf. Pt. 1: Figs. 8(a) and 8(b)]; conversely, the largest distortions occur when the basis functions poorly approximate spectra [cf. Pt. 1: Figs. 8(c) and (d), in which low-temperature spectra show exponential decreases within $[\Delta E]]$.

Spectral functions in violation of the a priori assumptions. - The unfold passband functions may be used to predict how potential source spectra will be treated by the unfold algorithm [Eq. (2)]. Of particular interest is how the algorithm responds to spectra that violate its assumptions. We consider here a pair of troublesome examples: spectra either distinctly narrower than the unfold bins $\left[\Delta E_{j}\right]$ or considerably broader than the assumed unfold interval $[\Delta E]$.

Spectral emission lines $f_{\lambda}(E)$ were excluded from the unfold algorithm (Pt. 1: Sec. III, and Table I). Yet, such spectra must be confronted for $z$-pinch plasmas of low atomic numbers, densities, and temperatures [46]. We model here one emission line of width $\delta E>0$, located at $E_{\lambda, K}$, wholly within the single unfold bin $\left[\Delta E_{K}\right]$ :

$$
f_{\lambda}(E) \equiv \begin{cases}\mathcal{F}_{\lambda}(\delta E)^{-1} & \text { for }\left|E-E_{\lambda, K}\right| \leq \frac{1}{2}(\delta E) \\ 0 & \text { otherwise }\end{cases}
$$

where $\mathcal{F}_{\lambda}$ is the total $\mathrm{x}$-ray flux in the line, bin $K=1, \ldots$, or $N$, and $\left|E_{\lambda, K}-E_{K-1}\right|,\left|E_{\lambda, K}-E_{K}\right| \geq \frac{1}{2}(\delta E)>0$. The line width $(\delta E)$ is chosen sufficiently small that $\int f_{\lambda}(E) R_{i}(E) d E \approx \mathcal{F}_{\lambda} R_{i}\left(E_{\lambda, K}\right)$ over practical integration intervals containing $E_{\lambda, K}$. For this model, the unfold coefficients $f_{\lambda, j}$ come directly from Eq. (32) and $\left\langle f_{\lambda}\right\rangle_{j}=$ $\delta_{j, K}\left(\Delta E_{K}\right)^{-1} \mathcal{F}_{\lambda}$ :

$$
f_{\lambda j} \approx\left\langle f_{\lambda}\right\rangle_{j}+\left(\mathcal{F}_{\lambda}\right)\left[\rho_{U, j}\left(E_{\lambda, K}\right)-\frac{\delta_{j K}}{\Delta E_{K}}\right] .
$$

Equation (38) highlights two serious difficulties posed by line spectra: (a) $f_{\text {unfold }}(E)$ can, in general, be nonzero (even alternating in sign) in unfold bins that do not actually contain the spectral line [cf. Fig. 3(b)]; and, more importantly, (b) the binwise distortion, $f_{\lambda j}-\left\langle f_{\lambda}\right\rangle_{j}$, is a strong function of $E_{\lambda, K}$ via $\rho_{U, j}\left(E_{\lambda, K}\right)$. In particular, within $\left[\Delta E_{K}\right]$ (which by assumption contains the line) the unfold does not recover $f_{\lambda}\left(E_{\lambda, K}\right)$ or even $f_{\lambda}\left(E_{\lambda, K}\right)(\delta E)\left(\Delta E_{K}\right)^{-1}$, a result of significance for superposed continua and emission lines. Such difficulties with line spectra are common to all diagnostics with broad, overlapping, and energy-dependent response functions-especially, if $E_{\lambda, K}$ is unknown. Potential mitigating strategies include the removal of spectral lines from the continuum with suitable background channels [47], or the addition of an emission-line model $[15,48,49]$ to the basis functions-given $E_{\lambda, K}$ from ancillary measurements.

A second generic spectrum that violates the a priori assumptions of the unfold algorithm is a function $f_{\text {wide }}(E)$ that extends significantly beyond the assumed unfold interval, $[\Delta E]=\left[E_{\mathrm{LO}}, E_{\mathrm{HI}}\right]$ : i.e., $\int_{0}^{E_{\mathrm{MAX}}} f_{\text {wide }}(E) d E \gg \int_{E_{\mathrm{LO}}}^{E_{\mathrm{HI}}}$ $f_{\text {wide }}(E) d E$. For simplicity, we model $f_{\text {wide }}(E)$ as a general function outside $[\Delta E]$, but as a histogram within $[\Delta E]$ :

$$
f_{\text {wide }}(E) \equiv \begin{cases}f_{L}(E) & \text { for } 0 \leq E<E_{\mathrm{LO}} \\ \sum_{j=1}^{N} \varphi_{j} B_{j}(E) & \text { for } E_{\mathrm{LO}} \leq E<E_{\mathrm{HI}} \\ f_{H}(E) & \text { for } E_{\mathrm{HI}} \leq E<E_{\mathrm{MAX}}\end{cases}
$$

where $f_{L}(E), f_{H}(E), \varphi_{j}>0$. It follows from this definition and Eq. (36) that

$$
\begin{aligned}
f_{\text {wide }, j}= & \left\langle f_{\text {wide }}\right\rangle_{j}+\int_{0}^{E_{\mathrm{LO}}} \rho_{U, j}(E) f_{L}(E) d E \\
& +\int_{E_{\mathrm{HI}}}^{E_{\mathrm{MAX}}} \rho_{U, j}(E) f_{H}(E) d E,
\end{aligned}
$$

where $\int_{E_{\mathrm{LO}}}^{E_{\mathrm{HI}}} \rho_{U, j}(E) \Delta f_{\mathrm{B}}(E) d E$ in Eq. (36) vanishes [50]. According to Eq. (40), $f_{\text {wide, } j}$ differs from $\left\langle f_{\text {wide }}\right\rangle_{j}$ due to the behavior of $f_{\text {wide }}(E)$ outside the unfold interval. For the responses here (Pt. 1: Fig. 2), $f_{L}(E)$ generally contributes little unfold distortion since $\rho_{U, j}\left(0 \leq E<E_{\mathrm{LO}}\right) \simeq 0$ [Fig. 3(b)]. But, a high-energy tail $f_{H}(E)$ can have a marked effect, particularly for the unfold in bins 4 and 5 , because both $\rho_{U, 4}(E)$ and $\rho_{U, 5}(E)$ show significant sensitivity out to $E_{\text {MAX }}$ [Fig. 3(b)]. Since $\left\langle f_{\text {wide }}\right\rangle_{4}=\varphi_{4}$ and $\left\langle f_{\text {wide }}\right\rangle_{5}=\varphi_{5}$ in this model, $f_{H}(E)$ makes $f_{\text {wide, } 4}<\varphi_{4}$ and $f_{\text {wide, } 5}>\varphi_{5}$, a particularly noticeable distortion if $\varphi_{4}, \varphi_{5} \cong 0$.

Negative unfold coefficients. - The last question posed in this subsection can also be addressed with the passband functions $\rho_{U, j}(E)$ in Fig. 3(b). The issue is this: How can the unfold operator $\mathcal{U}$ yield negative behavior in $f_{\text {unfold }}(E)$ when the response and spectral functions, $R_{i}(E)$ and $f(E)$ are non-negative? Such behavior is routinely encountered in the deconvolution of time series and image data, particularly when too "stiff" a compensation for nonideal frequency response and optical blurring is applied: negative temporal undershoots ("ringing") and spatial artifacts 
then occur $[3,7,26,51,52]$. From an abstract point of view, analogous effects obtain with the unfold algorithm: the inverse $\mathcal{M}_{\mathrm{BD}}^{-1}$ has too little flexibility to prevent negative behavior in the reconstructed spectrum. Here we (a) predict what negative unfold effects occur for arbitrary spectral functions and (b) trace their cause. How such negative behavior is treated in flux estimates $\mathcal{F}_{\text {unfold }}$ is discussed in the next subsection (Sec. III C).

According to Eqs. (32) and (33), the unfold coefficients $f_{j}$ are the inner products, $\left(\rho_{U, j} \mid f\right)=\int_{0}^{E_{\mathrm{MAX}}} \rho_{U, j}(E) \times$ $f(E) d E$. Thus, given $f(E) \geq 0$, values $f_{j}<0$ appear only if both $\rho_{U, j}(E)<0$ over some x-ray subinterval(s) of $\left[0, E_{\mathrm{MAX}}\right]$ and the shape of $f(E)$ allows such negative behavior to dominate $\int \rho_{U, j}(E) f(E) d E$. Now, because regions of negative $\rho_{U, j}(E)$ do occur in Fig. 3(b), a spectral candidate for producing $f_{j}<0$ will be either strongly peaked where $\rho_{U, j}(E)<0$ or largely averaged out in $\left[0, E_{\mathrm{MAX}}\right]$ except where $\rho_{U, j}(E)<0$. Hence, negative unfold coefficients can be predicted simply by comparing $f(E)$ with the $\rho_{U, j}(E)$ 's. For example, Fig. 3(b) and Planckian spectra $S_{b b}(E, T)$ (Pt. 1: Fig. 8 and Pt. 1: Table III) show that (a) enough of the high-energy exponential tail of $S_{b b}(E, T \geq 125 \mathrm{eV})$ extends above the $1020-\mathrm{eV}$ discontinuity in $\rho_{U, 4}(E)$ to make $S_{4}>0$, but that (b) this tail more heavily weights $\rho_{U, 4}(E)<0$ for lower- $T$ spectra, thereby yielding $S_{4}<0$. Nevertheless, since the negative behavior of $\rho_{U, j}(E)$ in Fig. 3(b) is both relatively shallow and spectrally confined, negative $f_{j}$ 's are generally not a serious problem for the responses and spectra studied (Pt. 1: Fig. 2 and Pt. 1: Table I). The Cauchy-Schwarz inequality [16-18] bounds the magnitude of these artifacts: $\left|f_{j}\right| \leq\left(\rho_{U, j} \mid \rho_{U, j}\right)^{1 / 2}(f \mid f)^{1 / 2}$. (Exceptions often point to other problems: e.g., in spectral approximation or calibration bias.)

What accounts for negative behavior of the unfold passband functions in this algorithm is spectrally overlapping responses. This conclusion follows from the non-negativity and independence of the response functions $R_{i}(E)$, our choice of basis functions, and the definition of the $\rho_{U, j}(E)$ 's [Eq. (33)]. That is, $\rho_{U, j}(E)$ yields negative values only if at least some of the elements $\left(\mathbb{R}^{-1}\right)_{j i}<0$. But, such negative elements of $\mathbb{R}$ must occur because $\mathbb{R}$ is non-

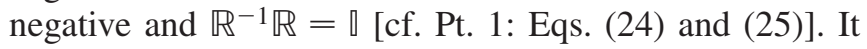
is thus the overlapping responses that produce the $R_{i j} \geq 0$ 's that ultimately lead to negative behavior in $\rho_{U, j}(E)$.

\section{Passband functions for the spectrally integrated $x$-ray flux}

Like the unfold coefficients $f_{j}$, the two scalar measures of integrated x-ray flux, $\mathcal{F}_{[\Delta E]}\left[\right.$ Eq. (6)] and $\mathcal{F}_{\text {unfold }}$ [Eq. (5)], may be expressed as inner products of arbitrary $f(E)$ with appropriate flux-passband functions, and $H_{[\Delta E]}(E)$ and $H_{\text {unfold }}(E)$. (Again, $\varepsilon_{i} \equiv 0$.)
The abstract tools for defining $H_{[\Delta E]}$ and $H_{\text {unfold }}$ are conceptually simpler than the operators $\mathcal{A}$ and $\mathcal{U}$ above. Required now are associations $\boldsymbol{h}[f]$ of $f(E)$ with scalars. Such mappings are called functionals over $\mathrm{L}_{2}$. In particular, we are here interested in linear functionals, for which $\boldsymbol{h}\left[f_{1}(E)+\lambda f_{2}(E)\right]=\boldsymbol{h}\left[f_{1}(E)\right]+\lambda \boldsymbol{h}\left[f_{2}(E)\right]$, where $\lambda$ is an arbitrary scalar.

In accord with Eq. (6), we define $\boldsymbol{h}_{[\Delta E]}[f]$ to associate $f(E)$ with its integral value $\mathcal{F}_{[\Delta E]}$ in the unfold interval. Thus, by $\boldsymbol{h}_{[\Delta E]}: \mathrm{L}_{2} \Rightarrow \Re$ we mean

$$
\boldsymbol{h}_{[\Delta E]}[f] \equiv \mathcal{F}_{[\Delta E]} \equiv \int_{E_{\mathrm{LO}}}^{E_{\mathrm{HI}}} f(E) d E=\left(H_{[\Delta E]} \mid f\right),
$$

where

$$
H_{[\Delta E]}(E) \equiv \sum_{j=1}^{N} B_{j}(E)= \begin{cases}1 & \text { if } E_{\mathrm{LO}} \leq E \leq E_{\mathrm{HI}} \\ 0 & \text { otherwise, }\end{cases}
$$

and $\Re$ is the field of real numbers. We call $H_{[\Delta E]}(E)$ in Eq. (42) the incident flux-passband function for $[\Delta E]$.

The second functional mapping $h_{\text {unfold }}[f]$ agrees with Eq. (5). That is, $f(E)$ is associated with $\mathcal{F}_{\text {unfold }}$. Schematically, $\boldsymbol{h}_{\text {unfold }}: L_{2} \Rightarrow L_{2}($ via $\mathcal{U}) \Rightarrow \Re$, where $\mathcal{U}$ is the previously defined unfold operator, represented by the passbands $\rho_{U, j}(E)$ [Eq. (33)]. Thus,

$$
\boldsymbol{h}_{\text {unfold }}[f] \equiv \mathcal{F}_{\text {unfold }} \equiv \int_{E_{\mathrm{LO}}}^{E_{\mathrm{HI}}} f_{\text {unfold }}(E) d E=\left(H_{\text {unfold }} \mid f\right),
$$

where

$$
H_{\text {unfold }}(E) \equiv \sum_{j=1}^{N}\left[\Delta E_{j}\right] \rho_{U, j}(E)=\sum_{j=1}^{N} \sum_{i=1}^{N} \Delta E_{j}\left(\mathbb{R}^{-1}\right)_{j i} R_{i}(E),
$$

which may be verified by substitution into Eq. (43) [cf. Eqs. (5)]. We call $H_{\text {unfold }}(E)$ the unfolded fluxpassband function.

Figure 4 compares $H_{[\Delta E]}(E)$ with $H_{\text {unfold }}(E)$. At x-ray energies $E$ above and below the unfold interval, $[\Delta E]=$ $\left[E_{\mathrm{LO}}, E_{\mathrm{HI}}\right]$, the flux-passband functions are similar: $H_{[\Delta E]}$ is zero by definition; $H_{\text {unfold }}$ is effectively zero below $[\Delta E]$ by x-ray attenuation and decreases monotonically above $[\Delta E]$. But, inside $[\Delta E], H_{[\Delta E]}(E)$ and $H_{\text {unfold }}(E)$ are significantly different: $H_{[\Delta E]}(E)$ is constant with value 1 but $H_{\text {unfold }}(E)$ oscillates about it. These oscillations again result from the characteristic x-ray edges in the responses functions [Eq. (44)].

Yet, despite pointwise differences, $H_{[\Delta E]}(E)$ and $H_{\text {unfold }}(E)$ share certain integral properties (Appendix E). Figure 5(a) shows the difference, $\Delta H(E) \equiv H_{\text {unfold }}(E)-$ $H_{[\Delta E]}(E)$, from which it can be shown that the oscillations of $H_{\text {unfold }}(E)$ about $H_{[\Delta E]}(E)$ average out within $[\Delta E]$, so that, in fact, 


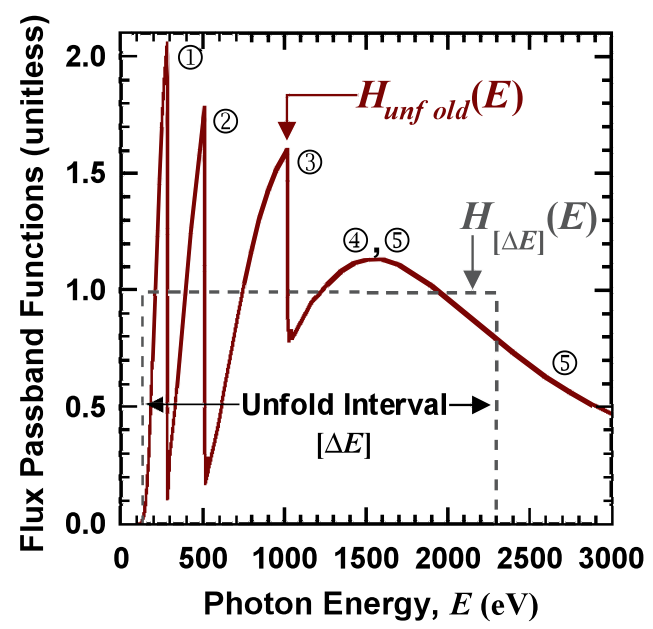

FIG. 4. Passband functions $H_{[\Delta E]}(E)$ and $H_{\text {unfold }}(E)$ for the incident flux $\mathcal{F}_{[\Delta E]}$ and unfolded flux estimate $\mathcal{F}_{\text {unfold }}$, respectively. The encircled numbers indicate roughly where specific response functions (Pt. 1: Fig. 1) dominate $H_{\text {unfold }}(E)$.

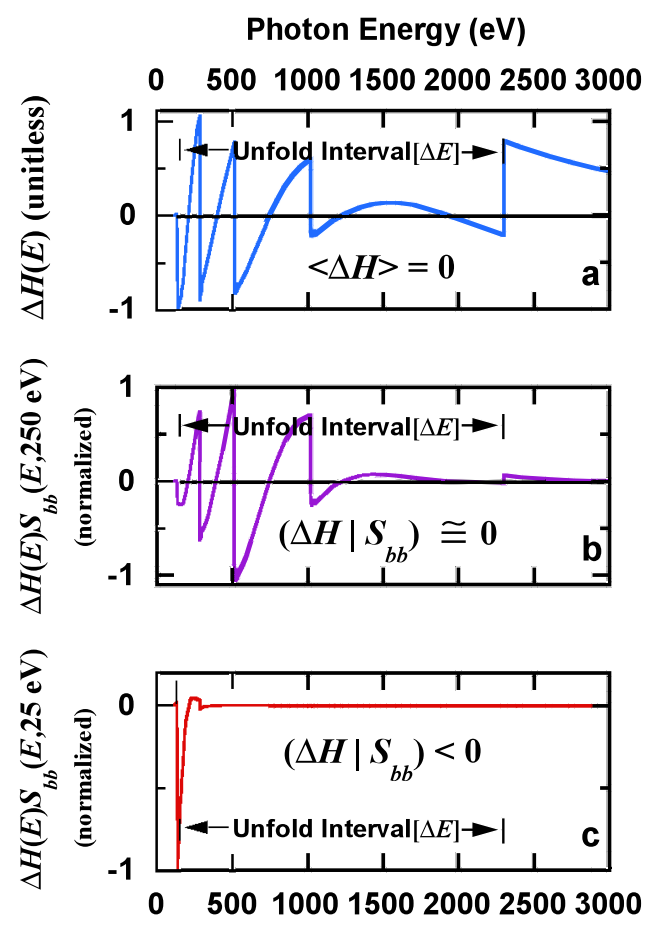

FIG. 5. Products of $\Delta H(E)$ with various Planckian spectra. Part (a) plots $\Delta H(E)$ alone, while (b) and (c) show the products, $\Delta H(E) S_{b b}(E, 250 \mathrm{eV})$ and $\Delta H(E) S_{b b}(E, 25 \mathrm{eV})$, respectively.

$$
\left\langle H_{\text {unfold }}\right\rangle=\left\langle H_{[\Delta E]}\right\rangle=1 .
$$

A similar result obtains for $\left(H_{[\Delta E]} \mid f\right)$ and $\left(H_{\text {unfold }} \mid f\right)$ if $f(E)$ is well approximated by the basis functions:

$$
\mathcal{F}_{\text {unfold }}=\left(H_{\text {unfold }} \mid f\right) \simeq\left(H_{[\Delta E]} \mid f\right)=\mathcal{F}_{[\Delta E]} .
$$

(These results are analogous to the integral properties of unfold passband functions $\rho_{U, j}$ [Sec. III B].) $\Delta H(E)$ in fact quantifies the disagreement, $\mathcal{F}_{\text {unfold }}-\mathcal{F}_{[\Delta E]}$ [Appendix E]:

$$
\begin{aligned}
\mathcal{F}_{\text {unfold }}-\mathcal{F}_{[\Delta E]} & =(\Delta H \mid f) \\
& =\int_{0}^{E_{\mathrm{MAX}}} H_{\text {unfold }}(E) \Delta f_{B}(E) d E
\end{aligned}
$$

[Eq. (34)]. \{This result is analogous to the binwise unfold distortions, $f_{j}-\langle f\rangle_{j}$ [Eq. (36)].

The flux-passband formalism may now be applied to new questions about the unfold algorithm: (a) Can the previously studied flux-recovery trends be understood [cf. Pt. 1: Fig. 9(b)]? (b) What is the bias in $\mathcal{F}_{\text {unfold }}$ for the troublesome spectra previously defined [Eqs. (37) and (39)]? And (c) what justifies the implicit use of negative unfold coefficients in estimating $\mathcal{F}_{\text {unfold }}$ ?

The behavior of $\mathcal{F}_{\text {unfold }} \mathcal{F}_{[\Delta E]}^{-1}$ vs $T$ for Planckian spectra $S_{b b}(E, T)$ (Pt. 1: Sec. IVA) can be understood directly from $\left(\Delta H \mid S_{b b}\right)$. The analysis is depicted in Figs. 5(a)-5(c), where Fig. 5(a) shows the flux-passband difference function, $\Delta H=H_{\text {unfold }}-H_{[\Delta E]}$, and Figs. 5(b) and 5(c) plot the product functions, $\Delta H(E) S_{b b}(E, 250 \mathrm{eV})$ and $\Delta H(E) S_{b b}(E, 25 \mathrm{eV})$, respectively. By Eq. (47), $\left(\Delta H \mid S_{b b}\right)$ is $\mathcal{F}_{\text {unfold }}-\mathcal{F}_{[\Delta E]}$. In Fig. 5(b), $\Delta H S_{b b}$ oscillates about zero due to $\Delta H(E)$, and multiple cycles are visible because $S_{b b}(E, 250 \mathrm{eV})$ is broad relative to $[\Delta E]$ [cf. Pt. 1: Fig. 8(a)]. These oscillations average out in the inner product: hence, $\left(\Delta H \mid S_{b b}\right) \simeq 0$ and $\mathcal{F}_{\text {unfold }} \mathcal{F}_{[\Delta E]}^{-1} \simeq 1$. By contrast, in Fig. 5(c), $\Delta H S_{b b}$ yields primarily a negative-going spike just above $E_{\mathrm{LO}}(137 \mathrm{eV})$, due to the limited penetration of $S_{b b}(E, 25 \mathrm{eV})$ into $[\Delta E]$ [cf. Pt. 1: Fig. 8(d)]. In this case there is no zero averaging and $\left(\Delta H \mid S_{b b}\right)<0$ : hence, $\mathcal{F}_{\text {unfold }} \mathcal{F}_{[\Delta E]}^{-1}<1$. Both of these results agree with Pt. 1: Fig. 9(b). The reason why the recovery ratios tend to unity with increasing $T$ is clear from the right-hand side of Eq. (47): the basis-function approximation improves as such spectra spread out over $[\Delta E]$ (i.e., $\left.\Delta f_{B}(E) \rightarrow 0\right)$; the same approximation degrades at low spectral temperatures (i.e., $\left|\Delta f_{B}(E)\right| \gg 0$ ).

Similar conclusions apply to the troublesome spectral functions, $f_{\lambda}(E)$ and $f_{\text {wide }}(E)$, defined above [Eqs. (37) and (39)], which were excluded from the unfold assumptions as poorly approximated by the $B_{j}(E)$ 's. $f_{\lambda}(E)$ was too localized, while $f_{\text {wide }}(E)$ was too broad relative to $[\Delta E]$. According to Eq. (47), the flux disagreement for the emission line model $f_{\lambda}(E)$ is $\mathcal{F}_{\text {unfold }}-\mathcal{F}_{[\Delta E]}=\mathcal{F}_{\lambda} \times$ $H_{\text {unfold }}\left(E_{\lambda}\right)-\mathcal{F}_{\lambda}$, which by Fig. 4 is again a strong function of line energy $E_{\lambda}$ [cf. Eq. (38)]; this result also shows that prominent emission-line flux need not simply average in when combined with continuum spectra. In the same way, applying Eq. (47) to $f_{\text {wide }}(E)$, one finds $\mathcal{F}_{\text {unfold }}-$ $\mathcal{F}_{[\Delta E]}=\int_{0}^{E_{\mathrm{LO}}} H_{\text {unfold }}(E) f_{L}(E) d E+\int_{E_{\mathrm{HI}}}^{E_{\mathrm{MAX}}} H_{\text {unfold }}(E) \times$ $f_{H}(E) d E$, which makes $\mathcal{F}_{\text {unfold }}$ exceed $\mathcal{F}_{[\Delta E]}$ since $H_{\text {unfold }}(E) \geq 0$ (Fig. 4). This result quantifies the flux error 
introduced by misjudging the unfold interval and corresponds to Eq. (40).

A final issue for this analysis is our acceptance of negative unfold coefficients in $\mathcal{F}_{\text {unfold }}$. Specifically, our definition [Eq. (5)] counts all the coefficients $f_{j}$ in $f_{\text {unfold }}(E)=\sum_{j=1}^{N} f_{j} B_{j}(E)$ algebraically. The general formulation of $\mathcal{F}_{\text {unfold }}$ as $\left(H_{\text {unfold }} \mid f\right)$ in Eqs. (43) and (44), of course, embodies the same procedure. Now, a philosophical objection to this approach insists that any $f_{j}<0$ is unphysical, possibly unpredictable, and cannot be included in an estimate of flux. In answer to this charge, it is well to remember that a histogram reconstruction $S_{\text {unfold }}(E)$ was developed as an overall approximation to $S(E)$ for constructing integrals in Eqs. (1) and (5); closeness to pointwise values or even binwise averages is not guaranteed (cf. Pt. 1: Sec. III H). The unfold passband functions $\rho_{U, j}(E)$ developed above do, however, show that coefficients, $f_{j}<0$, are bounded artifacts of the algorithm which are readily predictable [Fig. 3(b)] and which largely appear in $[\Delta E]$ where the source spectrum is relatively small. But, more importantly, since the goal of the $Z$ diagnostic is to estimate $\int f(E) d E$ in $[\Delta E]$, we have shown above (a) that $\mathcal{F}_{\text {unfold }} \simeq \int f(E) d E$ because the artifactcausing functions $\rho_{U, j}(E)$ compensate for one another in the flux-passband function $H_{\text {unfold }}(E)$ [Fig. 4]; (b) that disagreement between $\mathcal{F}_{\text {unfold }}$ and $\int f(E) d E$ is quantitatively understood and readily predictable for arbitrary $f(E)$; (c) that this flux estimate is well supported by simulations with physically relevant spectra (Pt. 1: Sec. IV); and (d) that an added non-negativity constraint need not, in fact, yield smaller bias than $\mathcal{F}_{\text {unfold }}$ (Pt. 1: Sec. IVA).

\section{SUMMARY AND CONCLUDING REMARKS}

The purpose of this article (in two parts) has been to characterize and evaluate an exactly determined, unfold algorithm for measuring spectrally integrated $\mathrm{x}$-ray flux incident on a calibrated filtered-XRD array. Part 1 described the formulation and testing of the algorithm. This second part considers error propagation due to deterministic perturbations (e.g., baseline bias and calibrational drift) and random errors (e.g., data noise and calibrational uncertainties). Significant sensitivity to response-function shifts was found in agreement with Gorbics [15], a result that argues for frequent calibrations of the detectors and filters. The effects of data noise and calibrational uncertainty were comparable in magnitude. It was also found that the algorithm amplified the noise-to-signal ratio (NSR) of input errors (noise and uncertainties) in estimating the reconstructed spectrum $S_{\text {unfold }}$, but reduced the NSR for $\mathcal{F}_{\text {unfold }}$. Comparisons of two independent filtered-XRD arrays in repeated experiments support the estimated uncertainty in $\mathcal{F}_{\text {unfold }}$. This second part also generalizes the data-simulation/unfold process from part 1 to include arbitrary input spectra by means of unfold and fluxpassband functions. These tools were used to understand the behavior of simulations in part 1 and to assess the effects of violations to the unfold assumptions (e.g., emission line spectra and continua that extend beyond the nominal unfold domain). The passband functions explain and bound negative unfold behavior and show a cancellation of such effects in $\mathcal{F}_{\text {unfold }}$.

A few concluding remarks are worth emphasizing with respect to this unfolding technique: (1) The first-order, error-propagation method derived in Sec. II (Appendix A) was designed to avoid an evaluation of $\Delta\left(\mathbb{R}^{-1}\right)\left[\neq(\Delta \mathbb{R})^{-1}\right]$ in Eq. (2). But, just as more sophisticated regularization methods of treating spectral inversion exist $[3,9,10,15,25-$ $28,35,39-41,48,49,53]$, so too more advanced statisticalinference tools weigh spectral distortion and bias against overall variance in the fit $[3,28,53-55]$. (2) The key to obtaining passband functions [Eqs. (31) and (33)] here is the separable form [24] of the kernels, $A\left(E, E^{\prime}\right)$ and $U\left(E, E^{\prime}\right)$, and can be obtained for unfold algorithms reducible to matrix inversion [i.e., $\mathbf{f}=\mathbb{K}^{-1} \mathbf{D}$, cf. Eq. (4), with $\mathbb{K}$ well conditioned], including some least-squares-based algorithms [9]. Twomey [56] has described such a reduction for the iterated Landweber algorithm. (3) The passband functions predict and bound the appearance of negative unfold coefficients. Such coefficients result from overlap in the response functions, our choice of basis functions, and $a$ priori assumptions. Higher-order basis functions [57-59] yield smoother reconstructions yet may still produce negative unfold behavior, unless a non-negativity constraint is imposed. Such a constraint need not decrease bias in the unfolded flux estimate [Pt. 1: Fig. 10(b); Pt. 1: Sec. IVA]. (4) Emission lines and spectra significantly broader than the assigned unfold interval are not well suited to the unfold algorithm given here. This conclusion follows from detailed studies of the passband functions, $\rho_{U, j}(E)$ and $H_{[\Delta E]}(E)$ [Secs. III B 2 and III C]. Line spectra produce unfold values remote to the location $E_{\lambda}$ of each line and are strongly dependent on $E_{\lambda}$ : the passband functions also predict bin-to-bin unfold oscillations for certain line spectra, as previously noted [57,60], and complicated flux averaging for continuum spectra with superposed prominent emission lines. (Background channels mitigate these effects $[47,61,62]$ by subtracting out the nettlesome lines.) Significantly broadened spectra distort the unfold reconstruction at high $\mathrm{x}$-ray energies: the passband functions bound such distortions. All these problems are endemic to $\mathrm{x}$-ray diagnostics with overlapping, low-resolution responses and are particularly acute if the photon energies involved are unknown. (5) We have employed the abstract structure in Sec. III primarily for its conceptual and simulation tools. This process was treated as noise free. \{Error propagation was considered separately so that the same formulas can apply to both real and simulated data [cf. Eq. (8) and Remark 6].\} But, data noise and calibrational 
uncertainties can be incorporated into the abstract formalism: the principal adjustment is to treat the $\varepsilon_{i}$ 's in Eq. (1) as an additional mapping $\mathcal{M}_{\varepsilon}$ of $\mathrm{D}$ into itself, inserted between $\mathcal{M}$ and $\mathcal{M}_{\mathrm{BD}}^{-1}$. [Such a mapping is consistent with both Eq. (8) and Monte Carlo analyses [6].] Under certain error models this adjustment yields uncertainties $\Delta \rho_{U, j}(E)$ in the unfold passband functions [Eq. (33)]. Further details are described in [63]. (6) We strongly advise potential users of the $Z$ diagnostic to study the passband functions, $\rho_{U, j}(E)$ and $H_{[\Delta E]}(E)$ [Figs. 3(b) and 4], as a quick check for compatibility between their anticipated experimental spectra and the unfold algorithm reported here. Quantitative distortions can be predicted from Eqs. (32) and (43). Such tests may be particularly useful if fine-structure spectra are anticipated. Settings for data recording can be predicted from Eq. (1). Unfold variances may be estimated from typical data noise and calibrational uncertainties by inserting the reconstruction of Eqs. (32) and (33) into Eqs. (11), (13), and (14) (Sec. II C); but, some of these equations may need adjustment, depending on the error and calibrational models chosen. (7) For those wishing to construct an unfold algorithm for their own filtered-XRD array, the following is a resume of the steps taken here: (a) The algorithm was formulated to answer issues raised in Pt. 1: Sec. III using representative response functions and a priori spectral information. (b) The algorithm was tested via simulations [Pt. 1: Sec. IV and Pt. 2: Sec. III, Eqs. (43) or (5)] and comparisons with other unfold techniques (Pt. 1: Sec. V). (c) During experiments, cable-compensated channel-voltage signals $V_{i}(t)$ were collected, reduced to channel data $D_{i}(t)$ (Pt. 1: Appendix A), and unfolded to yield the nominal unfold coefficients $S_{j}(t)$ of $S_{\text {unfold }}(t)$ and a flux estimate $\mathcal{F}_{\text {unfold }}(t)$ [Eqs. (2) and (5), respectively]; up-to-date response-function calibrations were important. (d) Overall uncertainty estimates, $\sigma\left(S_{j}\right)$ and $\sigma\left(\mathcal{F}_{\text {unfold }}\right)$, were added to the nominal $S_{\text {unfold }}(t)$ and $\mathcal{F}_{\text {unfold }}(t)$ : specifically, after characterization [Eqs. (11) and (D1) and Pt. 1: Appendix B], data noise and calibrational uncertainties were separately propagated in first order [Eqs. (17), (18), and (24)-(27), and Appendix D], then combined in quadrature [Sec. II C 3]. Dieck [64] discusses recommended methods of combining uncertainties in bias corrections with random error estimates. Some of these equations will need adjustment for differing error and calibrational models.

\section{ACKNOWLEDGMENTS}

This work was performed by Sandia National Laboratories. Sandia is a multiprogram laboratory operated by Sandia Corporation, a Lockheed Martin Company, for the United States Department of Energy under Contract No. DE-AC04-94AL85000. We also wish to thank the reviewers of this article for their patience, insights, and helpful suggestions.
APPENDIX A: DERIVATION OF $\Delta S$ AND $\Delta \mathcal{F}_{\text {unfold }}$ IN EQS. (8) AND (9)

We consider first data perturbations $\Delta D_{i}$ alone. Let a fixed spectrum $S(E)$ generate the noise-free data vector $\mathbf{d}$ [Eq. (1)]. If the response matrix $\mathbb{R}$ is well conditioned, the matrix equation, $\mathbb{R} \mathbf{S}=\mathbf{d}$, has a unique solution $\mathbf{S}$ [Eq. (4)]. Adding perturbations $\Delta D_{i}$ to $d_{i}$ changes the matrix equation to $\mathbb{R}(\mathbf{S}+\Delta \mathbf{S})=\mathbf{d}+\Delta \mathbf{D}=\mathbf{D}$, where $\Delta \mathbf{S}$ encompasses the change in unfold coefficients induced by $\Delta D_{i}$. Subtracting $\mathbb{R} \mathbf{S}=\mathbf{d}$ from this equation and inverting $\mathbb{R}$, one then finds $\Delta \mathbf{S}=\mathbb{R}^{-1}(\Delta \mathbf{D})$.

Next, consider perturbations $\Delta R_{i j}$ to the unfold-matrix elements $R_{i j}$ of $\mathbb{R}$. For convenience, let the matrix ( $\mathbb{R}+$ $\Delta \mathbb{R})$ correspond to responses, $R_{i}(E)+\Delta R_{i}(E)$, which interact with the fixed spectrum $S(E)$ to produce noise-free, channel data $\mathbf{d}$. A consistent unfold vector $\mathbf{S}$ is obtained by inverting $(\mathbb{R}+\Delta \mathbb{R}) \mathbf{S}=\mathbf{d}$. But, suppose that one unfolds $\mathbf{d}$ with $\mathbb{R}^{-1}$, instead of $(\mathbb{R}+\Delta \mathbb{R})^{-1}$ [both $\mathbb{R}$ and $\mathbb{R}+\Delta \mathbb{R}$ assumed invertible]. This inconsistent procedure yields a perturbed unfold, $\mathbf{S}+\Delta \mathbf{S}$, defined by $\mathbb{R}(\mathbf{S}+\Delta \mathbf{S})=\mathbf{d}$. Again, by subtraction, one isolates the perturbation $\Delta \mathbf{S}$ induced by the $\Delta R_{i j}$ 's: thus, $\mathbb{R}(\Delta \mathbf{S})=(\Delta \mathbb{R}) \mathbf{S}$ and $\Delta \mathbf{S}=$ $\mathbb{R}^{-1}[(\Delta \mathbb{R}) \mathbf{S}]$. Despite its formal complexity, $[(\Delta \mathbb{R}) \mathbf{S}]_{i}$ is equivalent to $\int_{0}^{E_{\mathrm{MAX}}} \Delta R_{i}(E) S(E) d E$ when $S(E)$ is approximated by $S_{\text {unfold }}(E)$, defined by unfold coefficients $(\mathbf{S})_{j}$ and basis functions $B_{j}(E)$.

In general, both of these generic perturbations independently contribute to $\varepsilon_{i}$, and the arguments for each can be combined to give Eq. (8) in the main text.

The effect of $\Delta \mathbf{S}$ on the unfolded flux estimate is likewise found from the definition, $\mathcal{F}_{\text {unfold }} \equiv \Delta \mathbf{E} \cdot \mathbf{S}$, where the fixed components of $\Delta \mathbf{E}$ are the unfold bin widths $\Delta E_{j}$. The same difference argument as above then yields $\Delta \mathcal{F}_{\text {unfold }}=\Delta \mathbf{E} \cdot(\Delta \mathbf{S})$, which on substitution gives Eq. (9). None of these expressions refer to a particular perturbation model (deterministic or random) for $\Delta \mathbf{D}$ or $\Delta \mathbb{R}$, examples of which are given in the main text.

\section{APPENDIX B: DERIVATION OF $\mathbb{C}(\Delta S)$ AND $\sigma^{2}\left(\Delta \mathcal{F}_{\text {unfold }}\right)$ IN EQS. (11) AND (12)}

Two general tools from probability theory apply to this analysis. The first links $\mathbb{C}(\Delta \mathbf{S})$ to $\Delta D_{i}$ and $\Delta R_{i j}$. That is, if $w\left(x_{1}, \ldots, x_{M}\right)$ and $z\left(x_{1}, \ldots, x_{M}\right)$ denote two functions of the random variables, $x_{1}, \ldots, x_{M}$, then the covariance $\operatorname{cov}(w, z)$ between $w$ and $z$ is given in first order [1] by

$$
\operatorname{cov}(w, z) \cong \sum_{i=1}^{M} \sum_{k=1}^{M}\left(\frac{\partial w}{\partial x_{i}}\right)\left(\frac{\partial z}{\partial x_{k}}\right) \operatorname{cov}\left(x_{i}, x_{k}\right),
$$

where all values of the indices are included and the derivatives are nonzero. Thus, from Eq. (8), one computes $\operatorname{cov}\left(\Delta S_{j}, \Delta S_{l}\right)=\sum_{i=1}^{N} \sum_{k=1}^{N}\left(\mathbb{R}^{-1}\right)_{j i} \operatorname{cov}\left(\Delta Y_{i}, \Delta Y_{k}\right)\left(\mathbb{R}^{-1}\right)_{l k}$, where $\Delta Y_{m}=\Delta D_{m}+\left([\Delta \mathbb{R}] \mathbf{S}_{\text {unfold }}\right)_{m}$ for $m=1, \ldots, N$. 
This is Eq. (13) in the text and may be more compactly written as Eq. (11).

The second useful probability theorem provides that if $y=y\left(x_{1}, \ldots, x_{M}\right)$ is a function of random variables $x_{1}, \ldots, x_{M}$, then to first order [1] one has

$$
\sigma^{2}(y)=\operatorname{cov}(y, y) \cong \sum_{j=1}^{M} \sum_{l=1}^{M}\left(\frac{\partial y}{\partial x_{j}}\right)\left(\frac{\partial y}{\partial x_{l}}\right) \operatorname{cov}\left(x_{j}, x_{l}\right),
$$

where all indices are again included in the double sum and the derivatives are nonzero. From Eq. (B2) and the definition of $\mathcal{F}_{\text {unfold }}$ in Eq. (5), one thus obtains the estimate $\sigma^{2}\left(\Delta \mathcal{F}_{\text {unfold }}\right)=\sum_{j=1}^{N} \sum_{l=1}^{N}\left(\Delta E_{j}\right) \operatorname{cov}\left(\Delta S_{j}, \Delta S_{l}\right)\left(\Delta E_{l}\right)$, listed as Eq. (14), which can also be written as Eq. (12) in the main text.

\section{APPENDIX C: VARIANCES, $\sigma^{2}\left(\Delta S_{j}\right)$ AND $\sigma^{2}\left(\Delta \mathcal{F}_{\text {unfold }}\right)$, FOR A RANDOM DATA-ERROR MODEL}

The error model for random noise perturbations $\Delta D_{i}$ posed in this example assumes no correlation of noise between channels $i$. The covariance matrix $\mathbb{C}(\Delta \mathbf{D})$, defined by Eq. (15), is then diagonal with eigenvalues, $\zeta_{i}^{2} d_{i}^{2}$, where $\zeta_{i}$ is the NSR, $\sigma\left(\Delta D_{i}\right) / d_{i}$.

Substituting $\mathbb{C}(\Delta \mathbf{D})$ into Eq. (11) and ignoring responsefunction perturbations, one finds the covariance matrix $\mathbb{C}(\Delta \mathbf{S})$ for unfold perturbations $\Delta S_{j}$ :

$$
\mathbb{C}(\Delta \mathbf{S})=\left(\mathbb{R}^{-1}\right)\left(\begin{array}{ccc}
\sigma^{2}\left(\Delta D_{1}\right) & \cdots & 0 \\
\vdots & \ddots & \vdots \\
0 & \cdots & \sigma^{2}\left(\Delta D_{N}\right)
\end{array}\right)\left(\mathbb{R}^{-1}\right)^{\mathbf{T}},
$$

the $j$, lth element of which is Eq. (16) from which Eq. (17) follows immediately.

The variance $\sigma^{2}\left(\Delta \mathcal{F}_{\text {unfold }}\right)$ is similarly estimated in this data-error model. Thus, one substitutes $\mathbb{C}(\Delta \mathbf{S})$ from Eq. (C1) into Eq. (12) to obtain

$$
\begin{aligned}
\sigma^{2}\left(\Delta \mathcal{F}_{\text {unfold }}\right)= & \Delta \mathbf{E} \cdot\left\{\left[\left(\mathbb{R}^{-1}\right)\left(\begin{array}{ccc}
d_{1}^{2} \zeta_{1}^{2} & \cdots & 0 \\
\vdots & \ddots & \vdots \\
0 & \cdots & d_{N}^{2} \zeta_{N}^{2}
\end{array}\right)\right.\right. \\
& \left.\left.\times\left(\mathbb{R}^{-1}\right)^{T}\right] \Delta \mathbf{E}\right\},
\end{aligned}
$$

which reduces to the triple sum,

$$
\sigma^{2}\left(\Delta \mathcal{F}_{\text {unfold }}\right)=\left\{\sum_{i=1}^{N} \sum_{j=1}^{N} \sum_{l=1}^{N} \Delta E_{j} \Delta E_{l}\left(\mathbb{R}^{-1}\right)_{j i}\left(\mathbb{R}^{-1}\right)_{l i} d_{i}^{2} \zeta_{i}^{2}\right\},
$$

and eventually simplifies to Eq. (18).
APPENDIX D: VARIANCES, $\sigma^{2}\left(\Delta S_{j}\right)$ AND $\sigma^{2}\left(\Delta \mathcal{F}_{\text {unfold }}\right)$, FOR A RANDOM CALIBRATIONAL ERROR MODEL

In the main text, Eqs. (21) and (22) translate fitparameter errors, $\Delta A_{i}$ and $\Delta \tau_{i}$, into errors $\Delta R_{i}(E)$ in the response functions. It is worth pointing out that this model works for filters and XRD's with multicomponents or chemical compounds only if the stochiometry is known; materials of unknown stochiometry require additional fit parameters (not considered here), yielding similar but more complicated results [cf. Pt. 1: Appendix B, Eqs. (B1) and (B2)].

The main text defines the elements $\Delta R_{i m}$ of $\Delta \mathbb{R}$ in Eq. (23). Statistical correlations, $\operatorname{cov}\left(\Delta R_{i j}, \Delta R_{k l}\right)$, between them are straightforwardly derived from Eq. (22) with the help of Appendix B, Eq. (B1). The result is

$$
\begin{aligned}
\operatorname{cov}\left(\Delta R_{i m}, \Delta R_{k n}\right)= & \delta_{i k}\left[\frac{\partial\left(\Delta R_{i m}\right)}{\partial\left(\Delta A_{i}\right)} \frac{\partial\left(\Delta R_{i n}\right)}{\partial\left(\Delta A_{i}\right)} \sigma^{2}\left(\Delta A_{i}\right)+0\right. \\
& \left.+0+\frac{\partial\left(\Delta R_{i m}\right)}{\partial\left(\Delta \tau_{i}\right)} \frac{\partial\left(\Delta R_{i n}\right)}{\partial\left(\Delta \tau_{i}\right)} \sigma^{2}\left(\Delta \tau_{i}\right)\right]
\end{aligned}
$$

where the zeros indicate terms that would correlate differing channels $i \neq k$ and $\Delta A_{i}$ with $\Delta \tau_{i}$, contrary to the assumptions of this error model. Performing the indicated operations, one finds

$$
\begin{aligned}
\operatorname{cov}\left(\Delta R_{i m}, \Delta R_{k n}\right)= & R_{i m} R_{i n}\left[\frac{\sigma^{2}\left(\Delta A_{i}\right)}{A_{i}^{2}}+\left\langle\tau_{i} \mu_{i, f}\right\rangle_{m}\right. \\
& \left.\times\left\langle\tau_{i} \mu_{i, f}\right\rangle_{n} \frac{\sigma^{2}\left(\Delta \tau_{i}\right)}{\tau_{i}^{2}}\right] \delta_{i k}
\end{aligned}
$$

The error model thus correlates only intrachannel matrix elements, $\Delta R_{i m}$ with $\Delta R_{i n}$, which share the same fit parameters, $A_{i}$ and $\tau_{i}$. [Compare Eq. (23) for averages of the form $\left\langle\tau_{i} \mu_{i, f}\right\rangle_{m}$.]

The next step is to calculate $\mathbb{C}[(\Delta \mathbb{R}) \mathbf{S}]$ from these covariances, coupled to the nominal unfold coefficients $S_{j}$ 's, which for error propagation are treated as constants. Now, $(\Delta \mathbb{R}) \mathbf{S}$ is a vector, the $i$ th component of which is $([\Delta \mathbb{R}] \mathbf{S})_{i}=\sum_{j=1}^{N}\left(\Delta R_{i j}\right) S_{j}$. Hence, the $i$, $k$ th element of $\mathbb{C}[(\Delta \mathbb{R}) \mathbf{S}]$ is $\operatorname{cov}\left\{([\Delta \mathbb{R}] \mathbf{S})_{i},([\Delta \mathbb{R}] \mathbf{S})_{k}\right\}$. To this, one again applies Eq. (B1) with the assumed independence of channels and finds that $\mathbb{C}[(\Delta \mathbb{R}) \mathbf{S}]$, like $\mathbb{C}(\Delta \mathbf{D})$ in Eq. (5), is diagonal:

$$
\mathbb{C}([\Delta \mathbb{R}] \mathbf{S})_{i k}=\delta_{i k} \sum_{m=1}^{N} \sum_{n=1}^{N} S_{m} S_{n} \operatorname{cov}\left(\Delta R_{i m}, \Delta R_{i n}\right)
$$

The covariances $\operatorname{cov}\left(\Delta R_{i m}, \Delta R_{i n}\right)$ are known from Eq. (D2): thus, 


$$
\begin{aligned}
\mathbb{C}([\Delta \mathbb{R}] \mathbf{S})_{i k}= & \delta_{i k} \sum_{m=1}^{N} \sum_{n=1}^{N} S_{m} S_{n} R_{i m} R_{i n}\left[\alpha_{i}^{2}+\left\langle\tau_{i} \mu_{i, f}\right\rangle_{m}\right. \\
& \left.\times\left\langle\tau_{i} \mu_{i, f}\right\rangle_{n} \theta_{i}^{2}\right],
\end{aligned}
$$

in which the NSR's $\left(\alpha_{i}\right.$ and $\left.\theta_{i}\right)$ of the calibrational fit parameters $\left(A_{i}\right.$ and $\tau_{i}$, respectively) are included.

The error-propagation process concludes by evaluating $\mathbb{C}(\Delta S)$ and $\sigma^{2}\left(\Delta \mathcal{F}_{\text {unfold }}\right)$. Equations (24) and (25) follow directly by substituting $\mathbb{C}([\Delta \mathbb{R}] \mathbf{S})$ into Eqs. (11) and (12), respectively [or, equivalently, into Eqs. (13) and (14)]. In these derivations we have also substituted $d_{i}$ for $\sum_{m=1}^{N} R_{i m} S_{m}$ and $\sum_{n=1}^{N} R_{i n} S_{n}$.

\section{APPENDIX E: INTEGRAL PROPERTIES OF $H_{[\Delta E]}(E)$ AND $H_{\text {unfold }}(E)$}

We begin by defining the difference function, $\Delta H(E) \equiv$ $H_{\text {unfold }}(E)-H_{[\Delta E]}(E)$, constructed from the flux-passband functions, $H_{\text {unfold }}(E)$ and $H_{[\Delta E]}(E)$ [Eqs. (42) and (44)], and plotted in Fig. 5(a). By direct computation, the unweighted average $\langle\Delta H\rangle$ of $\Delta H(E)$ over the unfold interval $[\Delta E]$ is

$$
\begin{aligned}
\langle\Delta H\rangle & =\left\langle H_{\text {unfold }}\right\rangle-\left\langle H_{[\Delta E]}\right\rangle \\
& =\frac{\int_{E_{\mathrm{LO}}}^{E_{\mathrm{HI}}}\left(\sum_{j=1}^{N}\left(\Delta E_{j}\right) \rho_{U, j}(E)-1\right) d E}{\Delta E}=0,
\end{aligned}
$$

a result that is visually consistent with Fig. 5(a) and yields Eq. (45).

Similarly, one forms the inner product of $\Delta H(E)$ with an arbitrary spectral function $f$. That is,

$$
\begin{aligned}
(\Delta H \mid f) & \equiv \int_{0}^{E_{\mathrm{MAX}}} \Delta H(E) f(E) d E \\
& =\left(H_{\text {unfold }} \mid f\right)-\left(H_{[\Delta E]} \mid f\right) .
\end{aligned}
$$

These inner products may be regarded as generalized moments of $H_{[\Delta E]}(E)$ and $H_{\text {unfold }}$ with fixed $f$. Equation (47) now follows by substituting the orthogonal decomposition of $f(E)$ from Eq. (34) and applying the same argument that led to Eq. (36). Thus, $(\Delta H \mid f)=\left(H_{\text {unfold }} \mid \sum_{k=1}^{N}\langle f\rangle_{k} B_{k}\right)+$ $\left(H_{\text {unfold }} \mid \Delta f_{B}\right)-\left(H_{[\Delta E]} \mid f\right)$; but, the first and third terms on the right-hand side cancel, leaving $(\Delta H \mid f)=$ $\left(H_{\text {unfold }} \mid \Delta f_{B}\right)=\int_{0}^{E_{\mathrm{MAX}}} H_{\text {unfold }}(E) \Delta f_{B}(E) d E$. If, in addition, $f$ is well approximated by the $B_{j}$ 's, then $\Delta f_{B}(E) \approx$ $0,\left(\Delta H \mid \Delta f_{B}\right) \approx 0$, and $\left(H_{\text {unfold }} \mid f\right) \approx\left(H_{[\Delta E]} \mid f\right)$, which is Eq. (46).

[1] S.L. Mayer, Data Analysis for Scientists and Engineers (Wiley, New York, 1975), p. 405f.

[2] H. H. Barrett and W. Swindell, Radiological Imaging: The Theory of Image Formation, Detection, and Processing (Academic Press, New York, 1981), Vol. 1.
[3] H.H. Barrett and K.J. Myers, Foundations of Image Science (Wiley-Interscience, Hoboken, NJ, 2004).

[4] G. A. Chandler, C. Deeney, M. Cuneo, D. L. Fehl, J. S. McGurn, R. B. Spielman, J. A. Torres, J. L. McKinney, J. Mills, and K.W. Struve, Rev. Sci. Instrum. 70, 561 (1999).

[5] Alternatively, if $\left\langle\Delta D_{i}\right\rangle$ and $\left\langle\Delta R_{i j}\right\rangle$ are nonzero, they may be treated as deterministic errors, and new random variables $\delta D_{i}$ and $\delta R_{i j}$ may be defined (e.g., $\delta D_{i} \equiv \Delta D_{i}-$ $\left\langle\Delta D_{i}\right\rangle$, etc.) for which $\left\langle\delta D_{i}\right\rangle=\left\langle\delta R_{i j}\right\rangle=0$.

[6] D. L. Fehl and F. Biggs, Rev. Sci. Instrum. 68, 890 (1997).

[7] W.H. Press, S. A. Teukolsky, W.T. Vetterling, B.P. Flannery, Numerical Recipes in FORTRAN: The Art of Scientific Computing (Cambridge University Press, New York, 1992), 2nd ed.

[8] D. L. Fehl, W. A. Stygar, G. A. Chandler, M. E. Cuneo, and C. L. Ruiz, Rev. Sci. Instrum. 76, 103504 (2005).

[9] I. J.D. Craig and J.C. Brown, Inverse Problems in Astronomy: A Guide to Inversion Strategies for Remotely Sensed Data (Adam Hilger Ltd., Boston, 1986).

[10] M. Bertero, C. De Mol, and E. R. Pike, Inverse Probl. 1, 301 (1985).

[11] R. Kress, Numerical Analysis (Springer, New York, 1998).

[12] G.E. Forsythe, M.A. Malcolm, and C.B. Moler, Computer Methods for Mathematical Computations (Prentice-Hall, Englewood Cliffs, NJ, 1977).

[13] D. S. Watkins, Fundamentals of Matrix Computations (Wiley, NY, 2002), 2nd ed.

[14] M. J. Maron, Numerical Analysis (Macmillan, New York, 1987), 2nd ed.

[15] S. G. Gorbics and N.R. Pereira, Rev. Sci. Instrum. 64, 1835 (1993).

[16] L. Råde and B. Westergren, Mathematics Handbook for Science and Engineering (Springer, New York, 2004), 5 th ed.

[17] I. N. Bronshtein and K. A. Semendyayev, Handbook of Mathematics (Springer, New York, 1998), 3rd ed.

[18] N.I. Akhiezer and I. M. Glazman, Theory of Linear Operators in Hilbert Space (Dover Publications, Mineola, NY, 1993).

[19] R. D. Evans, in Radiation Dosimetry, edited by F. H. Attix and W.C. Roesch (Academic Press, New York, 1968), 2nd ed.

[20] F. Reif, Fundamentals of Statistical and Thermal Physics (McGraw-Hill, New York, 1965).

[21] R. B. Bird, W. E. Stewart, and E. N. Lightfoot, Transport Phenomena (John Wiley and Sons, New York, 1960).

[22] D. Attwood, Soft X-rays and Extreme Ultraviolet Radiation (Cambridge University Press, Cambridge, UK, 1999).

[23] Let spectrum $S(E)$ generate noise-free channel data $\mathbf{d}$ and $\tilde{\mathbf{d}}$ in two sets of responses $\left\{R_{i}(E)\right\}$ and $\left\{\tilde{R}_{i}(E)\right\}$, respectively; and assume that corresponding matrices, $\mathbb{R}$ and $\tilde{\mathbb{R}}$, constructed from Eq. (3), are nonsingular. Then one can expect unfolds, $\mathbf{S}_{\text {unfold }}$ and $\tilde{\mathbf{S}}_{\text {unfold }}$, to satisfy Eq. (4) for the pairs $\mathbb{R}, \mathbf{d}$ and $\tilde{\mathbb{R}}, \tilde{\mathbf{d}}$, respectively. Using Eqs. (33), (34), and (36), coupled to the Cauchy-Schwarz inequality, one obtains $\left|S_{j}-\langle S\rangle_{j}-\left(\tilde{S}_{j}-\langle S\rangle_{j}\right)\right|=\mid \int_{0}^{E_{\operatorname{MAx}}}\left[\rho_{U, j}(E)-\right.$ $\tilde{\rho}_{U, j}(E) \Delta S_{\mathrm{B}}(E) d E \mid \leq\left\|\rho_{U, j}-\tilde{\rho}_{U, j}\right\|\left\|\Delta S_{\mathrm{B}}\right\|$, where $\left\|\rho_{U, j}\right\|$, $\left\|\tilde{\rho}_{U, j}\right\|,\left\|\tilde{\rho}_{U, j}-\tilde{\rho}_{U, j}\right\|<\infty$. If $S(E)$ is well approximated, $\left\|\Delta S_{B}\right\| \rightarrow 0$ and $S_{j} \rightarrow \tilde{S}_{j}$. 
[24] D.H. Griffel, Applied Functional Analysis (Dover Publications, Mineola, NY, 2002), originally published with revisions by Ellis Horwood Limited, Cichester, UK, 1985.

[25] C. de Mol, in Inverse Problems in Scattering and Imaging, Proceedings of a NATO Advanced Research Workshop, Cape Cod, USA, 1991, edited by M. Bertero and E. R. Pike (Adam Hilger, New York, 1992).

[26] M. Bertero and P. Boccacci, Introduction to Inverse Problems in Imaging (Institute of Physics Publishing, Philadelphia, 1998).

[27] R. Kress, Linear Integral Equations (Springer-Verlag, New York, 1999), 2nd ed.

[28] M. Bertero, C. De Mol, and E. R. Pike, Inverse Probl. 4, 573 (1988).

[29] M. Bertero, in Advances in Electronics and Electron Physics, edited by P.W. Hawkes (Academic Press, Harcourt Brace Jovanovich Publishers, Boston, 1989), Vol. 75, pp. 2-120.

[30] K. Rektorys, in Survey of Applicable Mathematics, edited by K. Rektorys (MIT Press, Cambridge, MA, 1969), p. 461.

[31] R. Courant and D. Hilbert, Methods of Mathematical Physics (Interscience, New York, 1953), Vol. 1, 1st ed.

[32] A. Kirsch, in Introduction to the Mathematical Theory of Inverse Problems (Springer, New York, 1996).

[33] A. N. Kolmogorov and S. V. Fomin, Introductory Real Analysis (Dover Publications, New York, 1975).

[34] N. Young, in Introduction to Hilbert Space (Cambridge University Press, Cambridge, UK, 1988).

[35] G. M. Wing, A Primer on Integral Equations of the First Kind: The Problem of Deconvolution and Unfolding (SIAM, Philadelphia, 1991).

[36] This type of operator generalizes the transformation of an $N$-dimensional vector $\mathbf{b}$ into another vector $\mathbf{b}^{\prime}$ via a matrix $\mathbb{T}:\left(\mathbf{b}^{\prime}\right)_{k}=(\mathbb{T} \mathbf{b})_{k}=\sum_{l=1}^{N} T_{k l} b_{l}$. In Eq. (29) the continuous variables, $E$ and $E^{\prime}$, in $K\left(E, E^{\prime}\right)$ correspond to rows and columns, respectively, in $\mathbb{T}$.

[37] D. L. Fehl, F. Biggs, G. A. Chandler, and W. A. Stygar, Rev. Sci. Instrum. 71, 3072 (2000).

[38] J.E. Monahan, in Scintillation Spectroscopy of Gamma Radiation, edited by S. M. Shafroth (Gordon and Beach, New York, 1967), Vol. 1, p. 371ff.

[39] G. E. Backus and J. F. Gilbert, Geophys. J. R. Astron. Soc. 13, 247 (1967).

[40] G. Backus and F. Gilbert, Geophys. J. R. Astron. Soc. 16, 169 (1968).

[41] G. Backus and F. Gilbert, Phil. Trans. R. Soc. A 266, 123 (1970).

[42] Substitution of the suggested $U\left(E, E^{\prime}\right)$ into Eq. (29) with rearranging yields $\mathcal{U}[f]=\sum_{j=1}^{N}\left[\sum_{i=1}^{N}\left(\mathbb{R}^{-1}\right)_{j i} \times\right.$ $\left.\int_{0}^{E_{\mathrm{MAX}}} R_{i}\left(E^{\prime}\right) f\left(E^{\prime}\right) d E^{\prime}\right] B_{j}(E)$, where the bracketed quantity is just $f_{j}$ from previous data simulations and unfolds_cf. Pt. 1, Eq. (27), with $S(E)$ replaced by $f(E)$. The bracketed quantity can be further rearranged as $\int_{0}^{E_{\operatorname{MAx}}}\left[\sum_{i=1}^{N}\left(\mathbb{R}^{-1}\right)_{j i} R_{i}\left(E^{\prime}\right)\right] f\left(E^{\prime}\right) d E^{\prime}$, which leads to Eqs. (32) and (33).

[43] This result follows from a direct integration of Eq. (33): $\int_{E_{k}}^{E_{k+1}} \rho_{U, j}(E) d E=\left(\rho_{U, j} \mid B_{k}\right)=\sum_{i=1}^{N}\left(\mathbb{R}^{-1}\right)_{j i} \times$ $\left(R_{i} \mid B_{k}\right)=\delta_{j k}$ because the inner-product terms $\left(R_{i} \mid B_{k}\right)$ are $R_{i k}=(\mathbb{R})_{i k}$, the summation yielding the $j, k$ th element of $\mathbb{R}^{-1} \mathbb{R}$.

[44] One rms measure of the relative closeness of a histogram, $\sum_{j=1}^{N} \beta_{j} B_{j}(E)$, to a given square-integrable spectral function $f(E)$ is $\min \left\|f-\sum_{j=1}^{N} \beta_{j} B_{j}\right\| /\|f\|$, where $\|*\|$ denotes the Euclidian norm over [0, $\left.E_{\mathrm{MAX}}\right]$. For the chosen basis histogram functions $B_{j}(E)$ [Pt. 1: Eq. (13)], this minimization yields $\beta_{j}=\langle f\rangle_{j}$, and the definition of closeness reduces to $\left(1-\sum_{j}^{N}\langle f\rangle_{j}^{2} \Delta E_{j} / \int_{0}^{E_{\mathrm{MAX}}} f^{2} d E\right)^{1 / 2}$, which is 0 if $f(E)$ is a similar histogram and 1 if $f(E)$ is orthogonal to all the $B_{j}(E)$ 's.

[45] Since the basis functions $B_{j}(E)$ are orthogonal, one can equate $f_{j}-\langle f\rangle_{j}$ with $\left(\rho_{U, j}-\rho_{A, j} \mid f\right)$ in Eq. (35). Substituting the decomposition of $f$ from Eq. (34) into this inner product and expanding, one has $\left(\rho_{U, j}-\right.$ $\left.\rho_{A, j} \mid \sum_{k=1}^{N}\langle f\rangle_{k} B_{k}+\Delta f_{\mathrm{B}}\right)=0+\left(\rho_{U, j} \mid \Delta f_{\mathrm{B}}\right)-0$, where the orthogonality of $\Delta f_{\mathrm{B}}$ to the $B_{k}$ 's has been used. Equation (35) is the definition of this quantity.

[46] J.P. Apruzese, J. Davis, K. G. Whitney, J. W. Thornhill, P. C. Kepple, R. W. Clark, C. Deeney, C. A. Coverdale, and T. W. L. Sanford, Phys. Plasmas 9, 2411 (2002).

[47] H. N. Kornblum, R. L. Kauffman, and J. A. Smith, Rev. Sci. Instrum. 57, 2179 (1986).

[48] R. Gold, Argonne National Laboratory Report No. ANL6984, TID-4500, Argonne National Laboratory, Argonne, IL, 1964, 37th ed.

[49] L. Kissel, F. Biggs, and T. R. Marking, Sandia National Laboratories Report No. SAND82-0396, Sandia National Laboratories, Albuquerque, NM, 1991.

[50] Within the $N$ bins $\left(\Delta E_{j}\right),\left\langle f_{\text {wide }}\right\rangle_{j} \equiv \varphi_{j}$. Hence, $\Delta f_{\mathrm{B}}(E)$, the portion of $f_{\text {wide }}(E)$ not approximated by the unfold basis functions $B_{j}(E)$ [Eq. (34)], is $f_{L}(E)$ for $E \leq E_{\mathrm{LO}}, 0$ for $E_{\mathrm{LO}}<E \leq E_{\mathrm{HI}}$, and $f_{H}(E)$ for $E_{\mathrm{HI}}<E$. Equation (40) then follows from Eq. (36).

[51] W. J. Randel, in Statistical Methods for Physical Science, Methods of Experimental Physics Vol. 28, edited by J. L. Stanford and S. B. Vardeman (Academic Press, New York, 1994).

[52] S. J. Howard, in Deconvolution of Images and Spectra, edited by P. A. Jansson (Academic Press, New York, 1984).

[53] P.C. Hansen, Discrete Inverse Problems: Insight and Algorithms, Fundamentals of Algorithms (SIAM, Philadelphia, 2010).

[54] D. B. Percival and A.T. Walden, Wavelet Methods for Time Series Analysis (Cambridge University Press, New York, 2000).

[55] T. Hastie, R. Tibshirani, and J. Friedman, The Elements of Statistical Learning: Data Mining, Inference, and Prediction (Springer-Verlag, New York, 2001).

[56] S. Twomey, Introduction to the Mathematics of Inversion in Remote Sensing and Indirect Measurements (Elsevier, New York, 1977), reprinted with corrections by Dover Press, Mineola, NY, 1996.

[57] Jing Li, Xian-Bin Huang, Si-Qun Zhang, Li Bing Yang, Wei-Ping Xie, and Yi-kang Pu, Rev. Sci. Instrum. 80, 063106 (2009).

[58] J. P. Knauer and N. C. Gindale, Rev. Sci. Instrum. 75, 3714 (2004). 
[59] C. De Boor, A Practical Guide to Splines, Applied Mathematical Science Series No. 27 (Springer, New York, 1978).

[60] D. L. Fehl, R. J. Leeper, and R. P. Kensek, Rev. Sci. Instrum. 63, 4786 (1992).

[61] R. L. Kaufman et al., Rev. Sci. Instrum. 66, 678 (1995).

[62] J. L. Bourgade, B. Villette, J. L. Bocher, J. Y. Boutin, S. Chiche, N. Dague, D. Gontier, J. P. Jadaud, B. Savale, R. Wrobel, and R.E. Turner, Rev. Sci. Instrum. 72, 1173 (2001).

[63] Assume that $\varepsilon_{i}$ can be modeled as $\left(\Delta \zeta_{i}\right) D_{i}+$ $\int_{0}^{E_{\mathrm{MAX}}} \Delta R_{i}(E) S(E) d E=\int_{0}^{E_{\operatorname{MAX}}}\left[\left(\Delta \zeta_{i}\right) R_{i}(E)+\Delta R_{i}(E)\right] \times$ $S(E) d E$, where $\Delta \zeta_{i}$ and $\Delta R_{i}(E)$ represent perturbations to the data and the response calibrations. In contrast to Appendix A, one may treat $\mathbb{R}$ in Eq. (2) as a given constant and immediately has $\Delta S_{j}=\sum_{i=1}^{N}\left(\mathbb{R}^{-1}\right)_{j i} \times$ $\int_{0}^{E_{\mathrm{MAx}}}\left[\left(\Delta \zeta_{i}\right) R_{i}(E)+\Delta R_{i}(E)\right] S(E) d E \quad$ and $\Delta \rho_{U, j}=$ $\sum_{i=1}^{N}\left(\mathbb{R}^{-1}\right)_{j i}\left[\left(\Delta \zeta_{i}\right) R_{i}(E)+\Delta R_{i}(E)\right]$, as in Eqs. (32) and (33). Error propagation now proceeds to $\sigma^{2}\left(\Delta \mathcal{F}_{\text {unfold }}\right)$ via $\operatorname{cov}\left(\Delta \rho_{U, j}, \Delta \rho_{U, l}\right) \quad$ and $\operatorname{cov}\left(\Delta S_{j}, \Delta S_{l}\right)$. The term $\int_{0}^{E_{\mathrm{MAX}}} \Delta R_{i}(E) S(E) d E$ above is the same as $(\Delta \mathbb{R} \mathbf{S})_{i}$ in Eqs. (8) and (9) and Appendix $C$ due to the collocation assumption for the unfold algorithm (Pt. 1: Sec. III E).

[64] R.H. Dieck, Measurement Uncertainty: Methods and Applications (Instrument Society of America, North Carolina, 1997), 2nd ed. 